Supporting Information

\title{
Assembly Transformation Jointly Driven by LAP Enzyme and GSH Boosting Theranostic Capability for Effective Tumor Therapy
}

Anna Wang, ${ }^{\ddagger}$ Jing Fang, ${ }^{\ddagger}$ Shuyue Ye, ${ }^{\ddagger}$ Qiulian Mao, ${ }^{\ddagger}$ Yan Zhao, ${ }^{\ddagger}$ Chaoxiang Cui, ${ }^{\ddagger}$

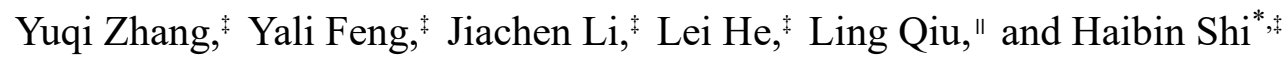

${ }^{\sharp}$ State Key Laboratory of Radiation Medicine and Protection, School for Radiological and Interdisciplinary Sciences (RAD-X) and Collaborative Innovation Center of Radiation Medicine of Jiangsu Higher Education Institutions, Soochow University, Suzhou 215123, P. R. China

"Laboratory of Nuclear Medicine, Ministry of Health, Jiangsu Key Laboratory of Molecular Nuclear Medicine, Jiangsu Institute of Nuclear Medicine, Wuxi 214063, P. R. China

Corresponding author*E-mail: hbshi@suda.edu.cn 


\section{Table of Contents}

\section{Experimental Procedures.}

1. Materials and instruments.

2. Flow cytometry analysis of cellular uptake.

3. Culture of 3D tumor spheres and penetration of nanoparticles.

4. Colocalization assay.

5. Cell viability assay.

6. Animal models.

7. Biodistribution.

8. Measurement of intracellular ROS production.

9. Lysosomal disruption

10. Evaluation of the effect of PDT treatment through Live/dead cell co-staining.

11. Cytotoxicity assay (MTT).

12. Flow cytometric analysis of cell apoptosis.

13. In vivo ROS production.

14. HE and TUNEL staining of tumorous tissues.

15. The detection of cell hypoxia.

16. Measurement of intracellular HIF-1a levels.

17. DNA double-strand breaks and cell migration assay.

18. Immunofluorescence staining for tumoral hypoxia evaluation.

19. HE and TUNEL staining of tumors received RT.

\section{Supplementary Schemes and Figures.}

Scheme S1. Synthetic route for compound Ce6-Leu and Ce6-Ac.

Figure S1. ${ }^{1} \mathrm{H}$ NMR spectrum of compound Ce6-3.

Figure S2. MALDI-TOF/MS spectrum of compound Ce6-3.

Figure S3. ${ }^{1} \mathrm{H}$ NMR spectrum of compound Ce6-2.

Figure S4. MALDI-TOF/MS spectrum of compound Ce6-2.

Figure S5. MALDI-TOF/MS spectrum of compound Ce6-Leu-Boc.

Figure S6. MALDI-TOF/MS spectrum of compound Ce6-Leu. 
Figure S7. MALDI-TOF/MS spectrum of compound Ce6-Ac.

Figure S8. The stability of Ce6-Leu.

Figure S9. MALDI-TOF/MS spectrum of compound Ce6-Cleaved.

Figure S10. MALDI-TOF/MS spectrum of compound Ce6-dimer.

Figure S11. Changes in fluorescence and particle size.

Figure S12. Confocal microscopy images of 3T3 cells and HepG2 cells incubated with the Ce6-Leu.

Figure S13. Cellular distribution of Ce6-Leu.

Figure S14. Z-stack CLSM images of HepG2-tumor cell spheroids treated with Ce6-Leu at different times.

Figure S15. Cell viability of $3 \mathrm{~T} 3$ and HepG2 cells incubated with different concentrations of Ce6-Leu and Ce6-Ac.

Figure S16. In vivo NIRF images of the mice bearing HepG2 tumor at different time after tail intravenous injection of Ce6-Leu or Ce6-Ac.

Figure S17. Fluorescence images and quantitative biodistribution analysis of major organs.

Figure S18. The blood circulation curve of Ce6-Leu.

Figure S19. UV-vis absorbance spectra of Ce6-Ac with or without treated with LAP and GSH.

Figure S20. The singlet oxygen generation ability of different agent.

Figure S21. The singlet oxygen generation ability of different agent in HepG2 cells.

Figure S22 DIC images of HepG2 cells after different experimental operations.

Figure S23. Photographs of representative mice captured on day $0,2,6,10$ and 14 post-treatment.

Figure S24. The average weight of various groups tumors after 14 days of different treatments.

Figure S25 Average body weights of mice 14 days after the treatments.

Figure S26. Slices of the major organs harvested from tumor-bearing mice receiving different treatments, stained hematoxylin and eosin (H\&E). 
Figure S27 MALDI-TOF/MS spectrum of compound Ce6-Leu@Mn+2+.

Figure S28 MALDI-TOF/MS spectrum of compound Ce6-Ac@Mn²+.

Figure S29. UV-vis absorbance spectra of Ce6-Ac and $\mathrm{Mn}^{2+}$ chelated Ce6-Ac.

Figure S30. T1-weighted MR images and longitudinal relaxivity $\left(r_{1}\right)$ of Ce6-Ac@Mn²+.

Figure S31. The toxicity of Ce6-Leu@Mn ${ }^{2+}$ and Ce6-Ac@Mn ${ }^{2+}$ to $3 T 3$ cells.

Figure S32. The detection of cell hypoxia.

Figure S33. The corresponding quantitative analysis of cell migration.

Figure S34. In vivo PA images of the tumor and the PA signals at different time points after intravenous injection of Ce6-Leu, Ce6-Ac@Mn+ or Ce6-Leu@Mn²+.

Figure S35. Immunofluorescence staining for tumoral hypoxia evaluation.

Figure S36. The mice photos of day 0, 2, 6, 10, 14 after different treatment.

Figure S37. The average weight of and average body weights of mice in each group. 


\section{Experimental Procedures}

\section{Materials and instruments}

All the starting chemicals were purchased from commercial suppliers and used without further purification, unless indicated otherwise. HPLC profiles were acquired by using 1260 high performance liquid chromatography (Agilent). UV-vis absorption spectra were taken on a UV spectrometer (UV-3600, Shimadzu). The fluorescence spectra were measured on an Edinburgh FLS980 spectrophotometer. Dynamic light scattering (DLS) measurements were performed on a particle size analyzer (Nano ZS90, Malvern). The in vivo fluorescence images were acquired using an IVIS spectrum imaging system (PerkinElmer). The confocal images were captured on a fluorescence microscope (FV1200, Olympus). PA imaging was performed with a Multispectral Optoacoustic Tomography scanner (MSOT, iThera medical, Germany). MR imaging was using a 3.0 T clinical MR scanner (MR solutions, UK).

2. Flow cytometry analysis of cellular uptake. HepG2 cells were seeded into 6-well plates at a density of $4 \times 10^{6}$ cells per well. After the growth in cell incubator for $12 \mathrm{~h}, \mathrm{HepG} 2$ cells were washed and treated with 20 $\mu \mathrm{M}$ Ce6-Leu or Ce6-Ac in a fresh culture medium for $8 \mathrm{~h}$ at $37^{\circ} \mathrm{C}$, respectively. Then all the cells were washed twice with cold PBS solution and then detached from the plate using a cell scraper. The collected cell pellets were eventually resuspended in cold PBS and transferred into a test tube for flow cytometry analysis.

3. Culture of 3D tumor spheres and penetration of nanoparticles. The multicellular spheroids were prepared according to previous reports. HepG2 cells were seeded into $1 \%$ agarose-coated 96 -well plates at a density of 500 cells per well and cultured. After one week, multicellular spheroids can form spontaneously and grow to about $600 \mu \mathrm{m}$ gradually. Finally, the established spheroids were transferred into a 12 -well plate and incubated in DMEM medium that contained Ce6-Leu or Ce6-Ac $(20 \mu \mathrm{M})$ for $8 \mathrm{~h}$, respectively. And then the tumor spheres were washed by PBS, resuspended in fresh medium and observed by CLSM.

4. Colocalization assay. HepG2 cell were treated with Ce6-Leu $(20 \mu \mathrm{M})$ for $8 \mathrm{~h}$, followed by incubation with Lyso-tracker, Golgi-tracker or Mito-tracker. After washing with PBS, cells were visualized on confocal laser scanning microscopy (CLSM). The corresponding Pearson correlation coefficient was calculated by ImageJ software.

5. Cell viability assay. Mouse fibroblast cell line $3 T 3$ and human hepatocellular carcinomas HepG2 were seeded into 96 well plates at density of $8 \times 10^{3}$ cells per well and were grown overnight. And then cells were incubated with different concentrations $(0,1,2,4,8,16,32,64$ and $128 \mu \mathrm{M})$ of Ce6-Leu or Ce6-Ac for $24 \mathrm{~h}$. Finally, the relative survival rate of the cells was determined by standard MTT method.

6. Animal models. Female athymic nude mice, $5-8$ weeks old, were inoculated with HepG 2 cells $\left(5 \times 10^{6}\right.$ cells per mouse) via a subcutaneous injection into their right thigh. To construct the subcutaneous HepG2 tumor model. All animals were randomly divided into groups before the experiment. All experiments are carried out in accordance with all relevant ethical regulations.

7. Biodistribution. For the ex vivo biodistribution study, HepG2 tumor-bearing mice $(\mathrm{n}=3)$ were intravenously injected with Ce6-Leu or Ce6-Ac $(100 \mu \mathrm{M}, 200 \mu \mathrm{L})$. Mice were sacrificed at $4 \mathrm{~h}$ after administration. The tissues, including the heart, liver, spleen, lung, kidney and tumor were harvested and the fluorescence images of these tissues were obtained by the IVIS Spectrum in vivo imaging system.

8. Measurement of intracellular ROS production. The ${ }^{1} \mathrm{O}_{2}$ production capacity of Ce6-Leu or Ce6-Ac in HepG2 cells was evaluated using two independent methods. For fluorescence imaging assay, HepG2 cells 
were seeded into a glass dish for $12 \mathrm{~h}$ and then the cells were treated with Ce6-Leu or Ce6-Ac $(20 \mu \mathrm{M})$. After $8 \mathrm{~h}$ of incubation at $37^{\circ} \mathrm{C}$, the medium was then removed, washed with fresh medium once, and $1 \mathrm{~mL}$ fresh medium containing DCFH-DA $(50 \mu \mathrm{M})$ was then added into the cells. After incubation at $37^{\circ} \mathrm{C}$ for another 30 $\mathrm{min}$, the cells were irradiated with a $660 \mathrm{~nm}$ laser $\left(150 \mathrm{~mW} / \mathrm{cm}^{2}\right)$ for $5 \mathrm{~min}$. After irradiation, the cells were washed with PBS for three times, and the fluorescence images from DCF were visualized on confocal laser scanning microscopy (CLSM).

For quantitative analysis, HepG2 cells were seeded into 96 -well plates at a density of $8 \times 10^{3}$ cells per well for $12 \mathrm{~h}$ and then the cells were treated with Ce6-Leu or Ce6-Ac $(20 \mu \mathrm{M})$. After $8 \mathrm{~h}$ of incubation at $37^{\circ} \mathrm{C}$, the medium was then removed, washed with fresh medium once, and $100 \mu \mathrm{L}$ fresh medium containing DCFHDA $(50 \mu \mathrm{M})$ was then added into the cells. After incubation at $37^{\circ} \mathrm{C}$ for another $30 \mathrm{~min}$, the cells were irradiated with a $660 \mathrm{~nm}$ laser $\left(150 \mathrm{~mW} / \mathrm{cm}^{2}\right)$ for $5 \mathrm{~min}$. After irradiation, the cells were washed with PBS for three times, and the fluorescence intensity from DCF were quantified by microplate reader.

9. Lysosomal disruption. HepG2 cells were seeded into a glass dish for $12 \mathrm{~h}$ and then the cells were treated with Ce6-Leu $(20 \mu \mathrm{M})$. After $8 \mathrm{~h}$ of incubation at $37^{\circ} \mathrm{C}$, the cells were washed by PBS buffer for three times. Afterward, the cells were treated with or without $660 \mathrm{~nm}$ irradiation $\left(150 \mathrm{~mW} / \mathrm{cm}^{2}\right)$ for 1 min followed by Hoechst 33342 and Lyso-tracker staining for $15 \mathrm{~min}$ at $37^{\circ} \mathrm{C}$. After three times of PBS rinsing, confocal laser scanning microscopy (CLSM) were employed to observe the intracellular distribution of the probe.

10 Evaluation of the effect of PDT treatment through Live/dead cell co-staining. HepG2 cells were seeded into 12-well plates for $12 \mathrm{~h}$ and then the cells were treated with Ce6-Leu or Ce6-Ac $(20 \mu \mathrm{M})$ and incubated at $37^{\circ} \mathrm{C}$ for $8 \mathrm{~h}$. Blank HepG2 cells were set as control. After incubation, the medium was removed, washed with PBS, and fresh culture medium was added. The cells were then irradiated with or without a $660 \mathrm{~nm}$ laser (150 $\mathrm{mW} / \mathrm{cm}^{2}$ ) for $5 \mathrm{~min}$. After another $8 \mathrm{~h}$ of incubation, the cells treated with different combinations of treatments were stained with live/dead staining kit and observed by fluorescence microscope.

11. Cytotoxicity assay (MTT). HepG2 cells were seeded into 96 -well plates at a density of $8 \times 10^{3}$ cells per well for $12 \mathrm{~h}$ and then the cells were treated with Ce6-Leu or Ce6-Ac $(20 \mu \mathrm{M})$ and incubated at $37^{\circ} \mathrm{C}$ for $8 \mathrm{~h}$. Blank HepG2 cells were set as control. After incubation, the medium was removed, washed with PBS, and fresh culture medium was added. The cells were then irradiated with or without a $660 \mathrm{~nm}$ laser $\left(150 \mathrm{~mW} / \mathrm{cm}^{2}\right)$ for $5 \mathrm{~min}$. After incubation for further $24 \mathrm{~h}$, the medium was removed, washed with PBS for two times and $100 \mu \mathrm{L}$ MTT solution were added into each well. After incubating for $3 \mathrm{~h}$ at $37^{\circ} \mathrm{C}$, the solution in wells was removed carefully, and the purple formazan crystals were dissolved by addition of $100 \mu \mathrm{L}$ DMSO. The absorbance (OD) of formazan at $490 \mathrm{~nm}$ in each well was recorded on a microplate reader.

12. Flow cytometric analysis of cell apoptosis. HepG2 cells were seeded into 12 well plates and incubated at $37^{\circ} \mathrm{C}$ for $12 \mathrm{~h}$. After removing the original medium, Ce6-Leu or Ce6-Ac $(20 \mu \mathrm{M})$ was added into HepG2 cells in fresh DMEM medium $(1 \mathrm{~mL})$ and incubated at $37^{\circ} \mathrm{C}$ for $8 \mathrm{~h}$. Blank HepG2 cells were set as control. After incubation, the medium was removed, washed with PBS, and fresh culture medium was added. The cells were then irradiated with or without a $660 \mathrm{~nm}$ laser $\left(150 \mathrm{~mW} / \mathrm{cm}^{2}\right)$ for $5 \mathrm{~min}$. After incubation for further 24 $\mathrm{h}$, removing the medium, the cells were trypsinized, collected, resuspended in $0.4 \mathrm{~mL}$ cold PBS, and stained with Annexin V-FITC/PI. After staining, the apoptotic cell was subjected to flow cytometry analysis.

13. In vivo ROS production. Tumor-bearing mice were injected with solutions of PBS, Ce6-Leu or Ce6-Ac $(200 \mu \mathrm{M}, 200 \mu \mathrm{L})$ via tail veins. 4 h post-injection, the mice were intratumorally injected with DCFH-DA solution $(50 \mu \mathrm{M}, 50 \mu \mathrm{L})$, followed by irradiation with or without a $660 \mathrm{~nm}$ laser for $10 \mathrm{~min}\left(0.15 \mathrm{~W} / \mathrm{cm}^{-2}\right)$. 
Then, the tumors from various groups of mice were harvested and examined with CLSM to observe the fluorescence of DCF oxidized by ROS within the tumor cells.

14. HE and TUNEL staining of tumorous tissues. Tumors were dissected from mice on the $24 \mathrm{~h}$ after different treatment and fixed in neutral buffered formalin (10\%). Then, the tumors were sliced into pieces with a thickness of $4 \mu \mathrm{m}$ for hematoxylin and eosin (H\&E) and TUNEL staining. Then the slices were observed by CLSM.

15. The detection of cell hypoxia. HepG 2 cells were seeded on glass-bottom dishes at a density $8 \times 10^{3}$ cells per well overnight. And then, after $4 \mathrm{~h}$ incubation of different reagent $(30 \mu \mathrm{M})$, a probe (Hypoxia Red Detection Reagent) for hypoxia was added into HepG2 cells, and they were cultured at $37^{\circ} \mathrm{C}$ under $5 \% \mathrm{CO}_{2}$ for $30 \mathrm{~min}$. The excess reagent was removed by washing three times with PBS. Then, the nucleus was stained by Hoechst 33342 for $15 \mathrm{~min}$, and then characterized by a confocal laser scanning microscopy. $\left(\mathrm{CLSM} ; \lambda_{\mathrm{ex}}=596 \mathrm{~nm}, \lambda_{\mathrm{em}}=670 \mathrm{~nm}\right)$.

16. Measurement of intracellular HIF-1 $\alpha$ levels. Cellular HIF-1 $\alpha$ levels were measured by western blot analysis. After $4 \mathrm{~h}$ incubation of different agents $(30 \mu \mathrm{M})$, HepG2 cells were washed with ice-cold PBS for 3 times and lysed using RIPA lysis buffer containing complete protease inhibitor. $60 \mu \mathrm{g}$ protein from each sample was resolved by SDS-PAGE and transferred onto a PVDF membrane. After blocking for $2 \mathrm{~h}$ with $5 \%$ skim milk, PVDF membrane were incubated with HIF-1 $\alpha$ antibody overnight at $4^{\circ} \mathrm{C}$ and then incubated with a corresponding secondary antibody conjugated with horseradish peroxidase for $2 \mathrm{~h}$ at room temperature. PVDF membrane were visualized with an ECL-plus detection system.

17. DNA double-strand breaks and Cell migration assay. For DNA double-strand breaks assay, HpeG2 cells were seeded into $35-\mathrm{mm}$ dishes by a density of $5 \times 10^{4}$ and incubated overnight. The cells were divided into four groups, that is, RT, Ce6-Leu+RT, Ce6-Ac@ $\mathrm{Mn}^{2+}+\mathrm{RT}$ and Ce6-Leu@ $\mathrm{Mn}^{2+}+\mathrm{RT}$. Different mediacontaining reagent (at a concentration of $30 \mu \mathrm{M}$ ) were added into HpeG2 cells. After $4 \mathrm{~h}$ incubation, the excess reagent was removed by washing three times with PBS. Then HpeG2 cells were treated with X-ray at doses of $6 \mathrm{~Gy}$. After treatment, the cells were fixed with $4 \%$ paraformaldehyde for $0.5 \mathrm{~h}$ and permeabilized with $1 \%$ Triton X-100 for $10 \mathrm{~min}$ to rupture the cell membrane, and then blocked with $5 \% \mathrm{BSA}$ for $1 \mathrm{~h}$ at $37^{\circ} \mathrm{C}$. After that, the fixed cells were incubated with $400 \mu \mathrm{L} \gamma$-H2AX antibody at $4^{\circ} \mathrm{C}$ overnight followed by incubation with the secondary antibody $\mathrm{Cy} 3$-labeled goat antirabbit $\operatorname{IgG}(\mathrm{H}+\mathrm{L})$ for $1 \mathrm{~h}$ at $37^{\circ} \mathrm{C}$ after being washed. Finally, the nuclei were stained with Hoechst 33342 and then examined using an Olympus confocal microscope (Olympus, Tokyo, Japan) for analyzing the red phospho-H2AX signals.

For cell migration assay, HpeG2 cells were seeded overnight in a 6-well plate at $4 \times 10^{5}$ cells per well. Upon reaching about $95 \%$ confluence, different media-containing reagent (at a concentration of $30 \mu \mathrm{M}$ ) were added into HpeG2 cells. After $4 \mathrm{~h}$ incubation, the excess reagent was removed by washing three times with PBS. Then HpeG2 cells were treated with X-ray at doses of 0 or $6 \mathrm{~Gy}$, and $1 \mathrm{~mL}$ sterile pipette tip was used to scrape the cell layer to form a gap. The cell migration was manually quantified by photographs of cells preand $120 \mathrm{~h}$-post incubation.

18. Immunofluorescence staining for tumoral hypoxia evaluation. Mice bearing HpeG2 tumors were randomly divided into 4 groups. After $6 \mathrm{~h}$ intravenous injection of PBS $(200 \mu \mathrm{L})$, Ce6-Leu $(200 \mu \mathrm{L}, 200 \mu \mathrm{M})$, Ce6-Ac@ $\mathrm{Mn}^{2+}(200 \mu \mathrm{L}, 200 \mu \mathrm{M})$ or Ce6-Leu@ $\mathrm{Mn}^{2+}(200 \mu \mathrm{L}, 200 \mu \mathrm{M})$, tumors were dissected from mice and sliced into pieces for HIF-1 $\alpha$ immunofluorescence staining. The slices were observed by CLSM.

19. HE and TUNEL staining of tumors received RT. HepG2 tumor-bearing mice $(n=3)$ were intravenously 
injected with PBS, Ce6-Leu, Ce6-Ac@Mn² or Ce6-Leu@ Mn ${ }^{2+}(200 \mu \mathrm{M}, 200 \mu \mathrm{L})$. X-ray irradiation (8 Gy) was applied in tumors $6 \mathrm{~h}$ postinjection. Tumors were dissected from mice on the $48 \mathrm{~h}$ after different treatment and fixed in neutral buffered formalin (10\%). Then, the tumors were sliced into pieces with a thickness of 4 $\mu \mathrm{m}$ for hematoxylin and eosin (H\&E) and TUNEL staining. Then the slices were observed by CLSM.

\section{Supplementary Schemes and Figures.}

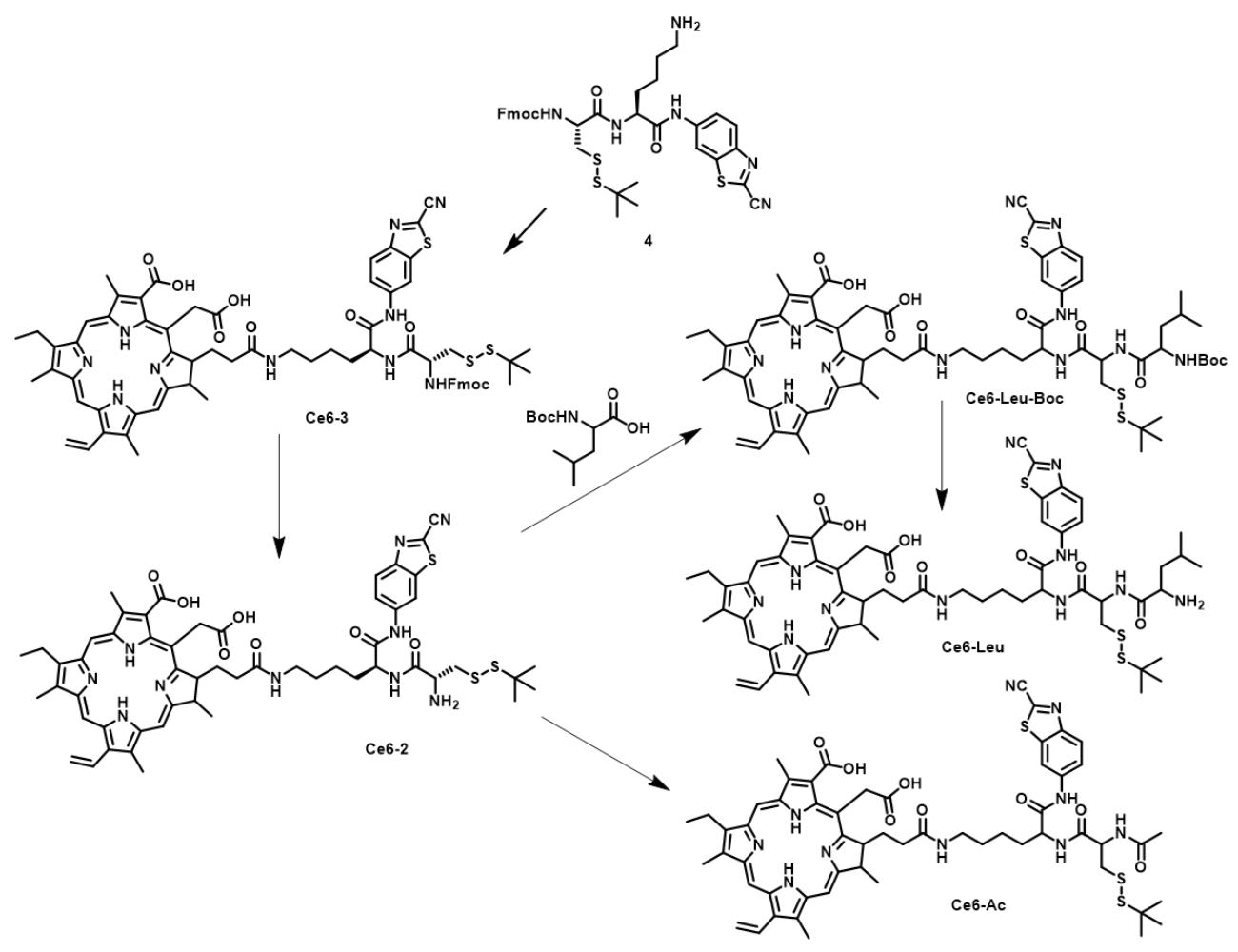

Scheme S1. Synthetic route for compound Ce6-Leu and Ce6-Ac.

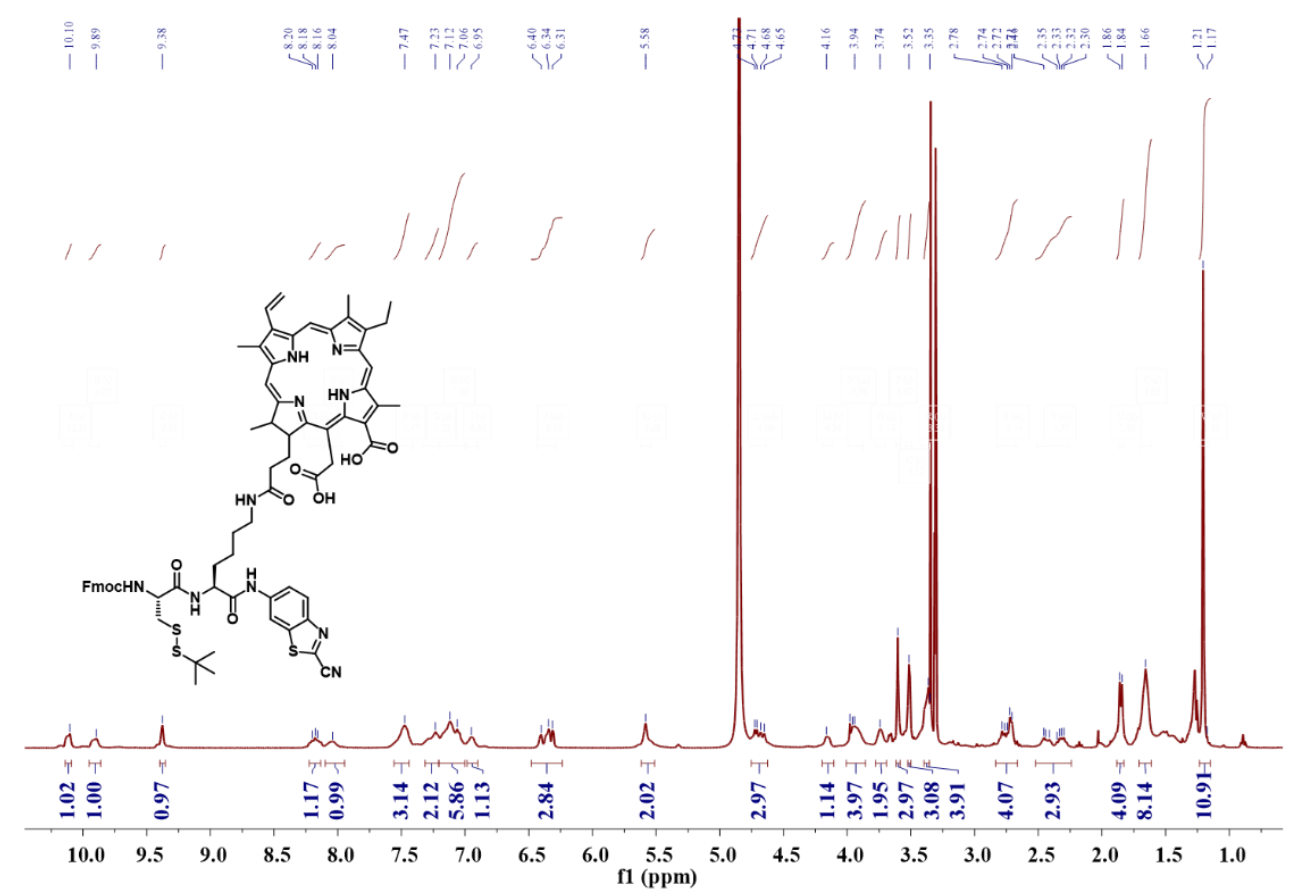


Figure S1. ${ }^{1} \mathrm{H}$ NMR spectrum of compound Ce6-3.

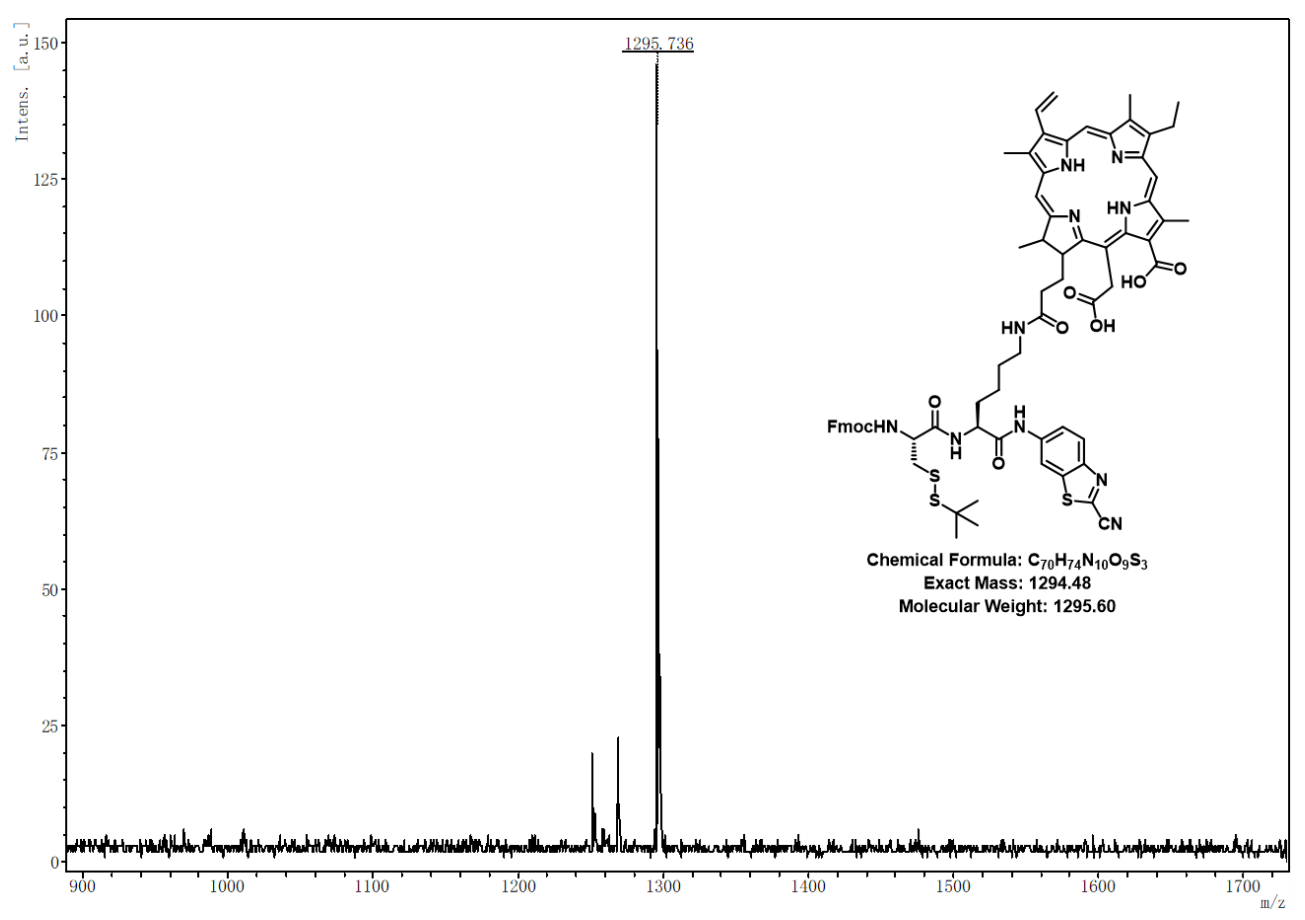

Figure S2. MALDI-TOF/MS spectrum of compound Ce6-3.

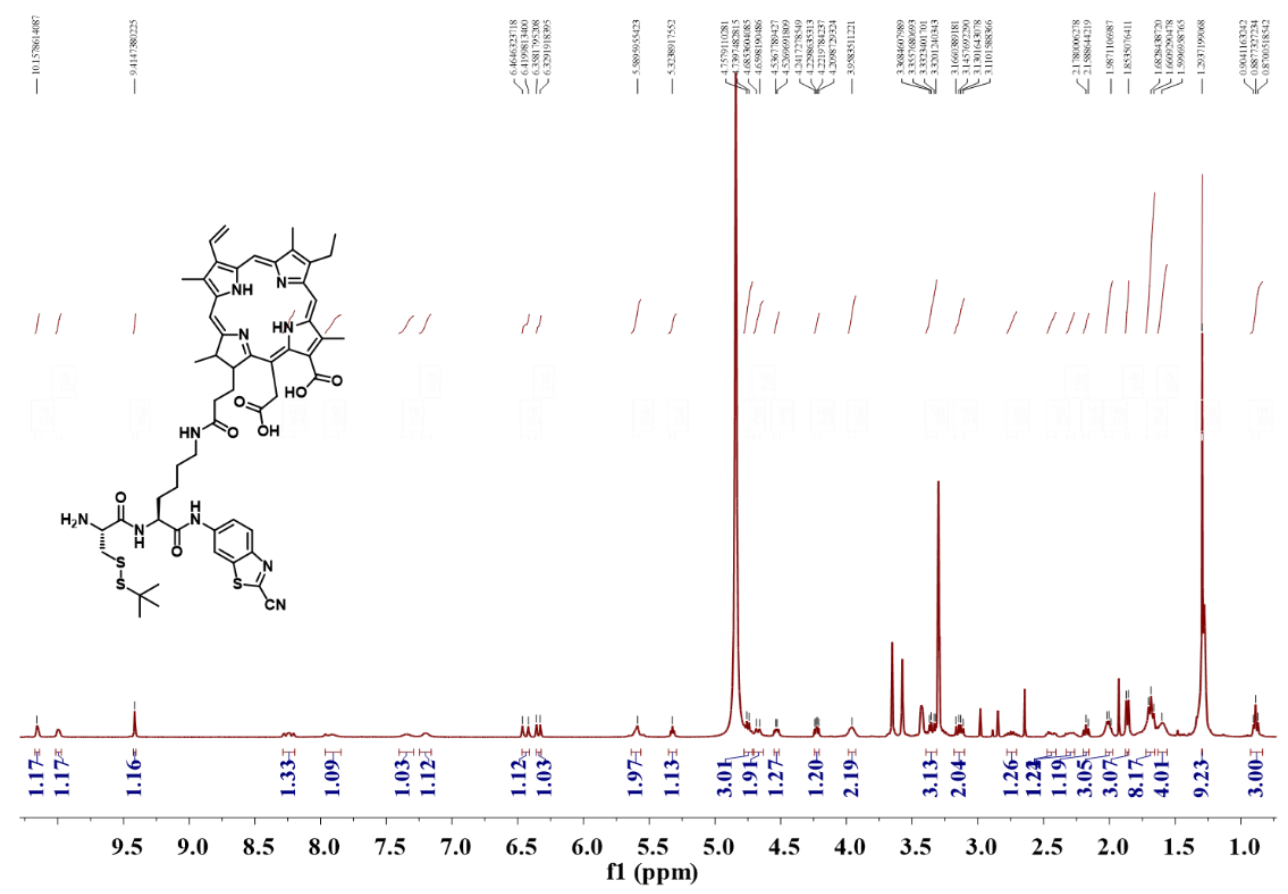

Figure S3. ${ }^{1} \mathrm{H}$ NMR spectrum of compound Ce6-2. 


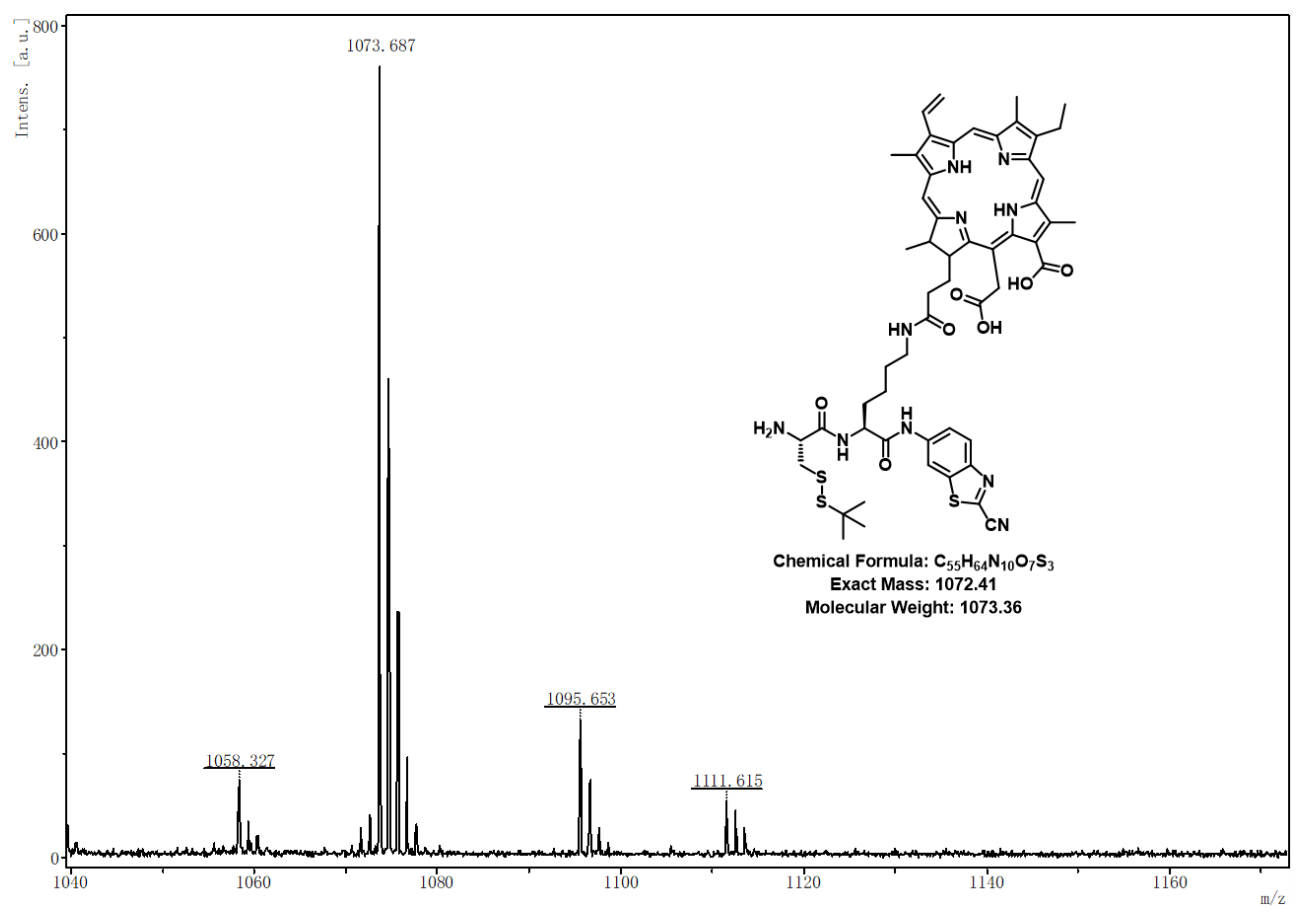

Figure S4. MALDI-TOF/MS spectrum of compound Ce6-2.

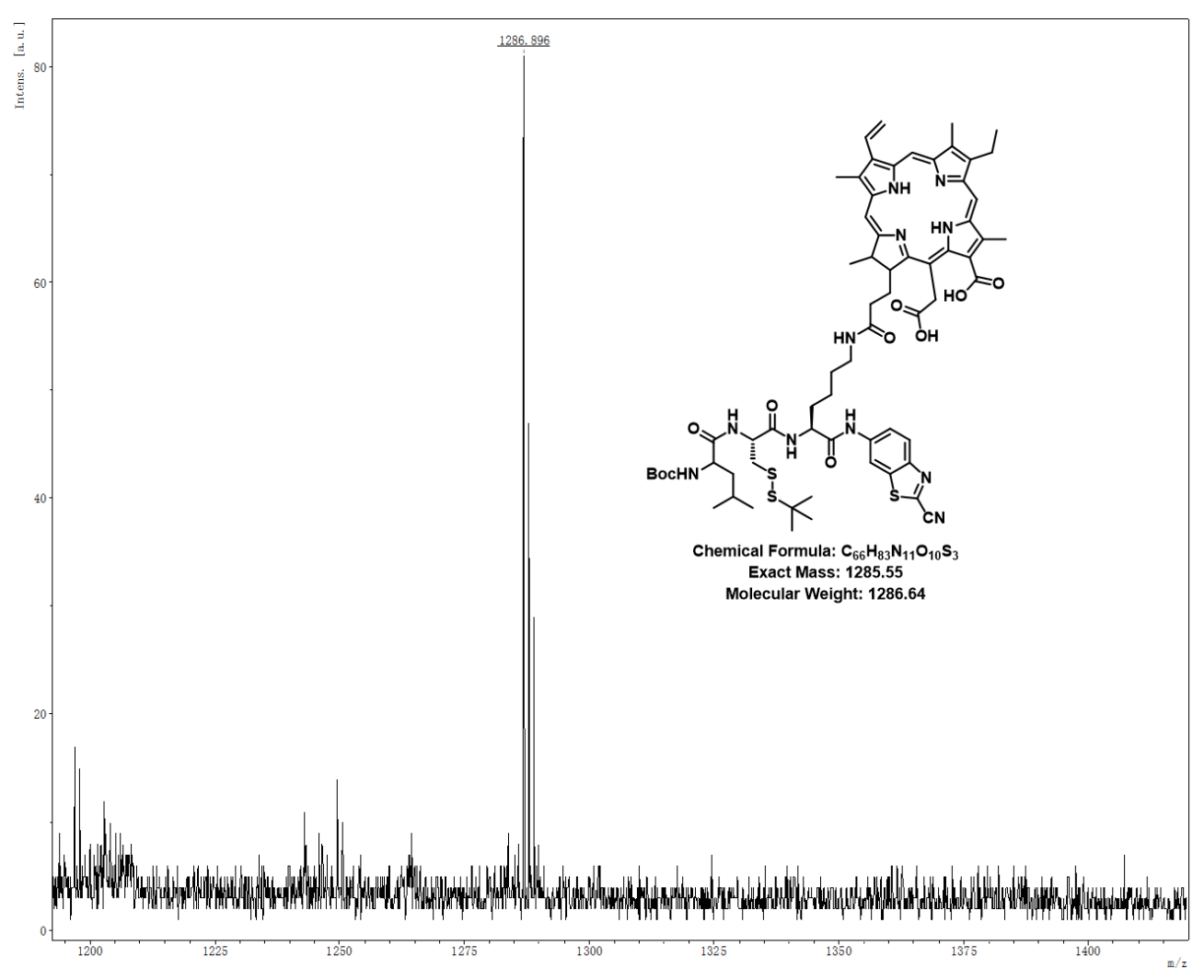

Figure S5. MALDI-TOF/MS spectrum of compound Ce6-Leu-Boc. 


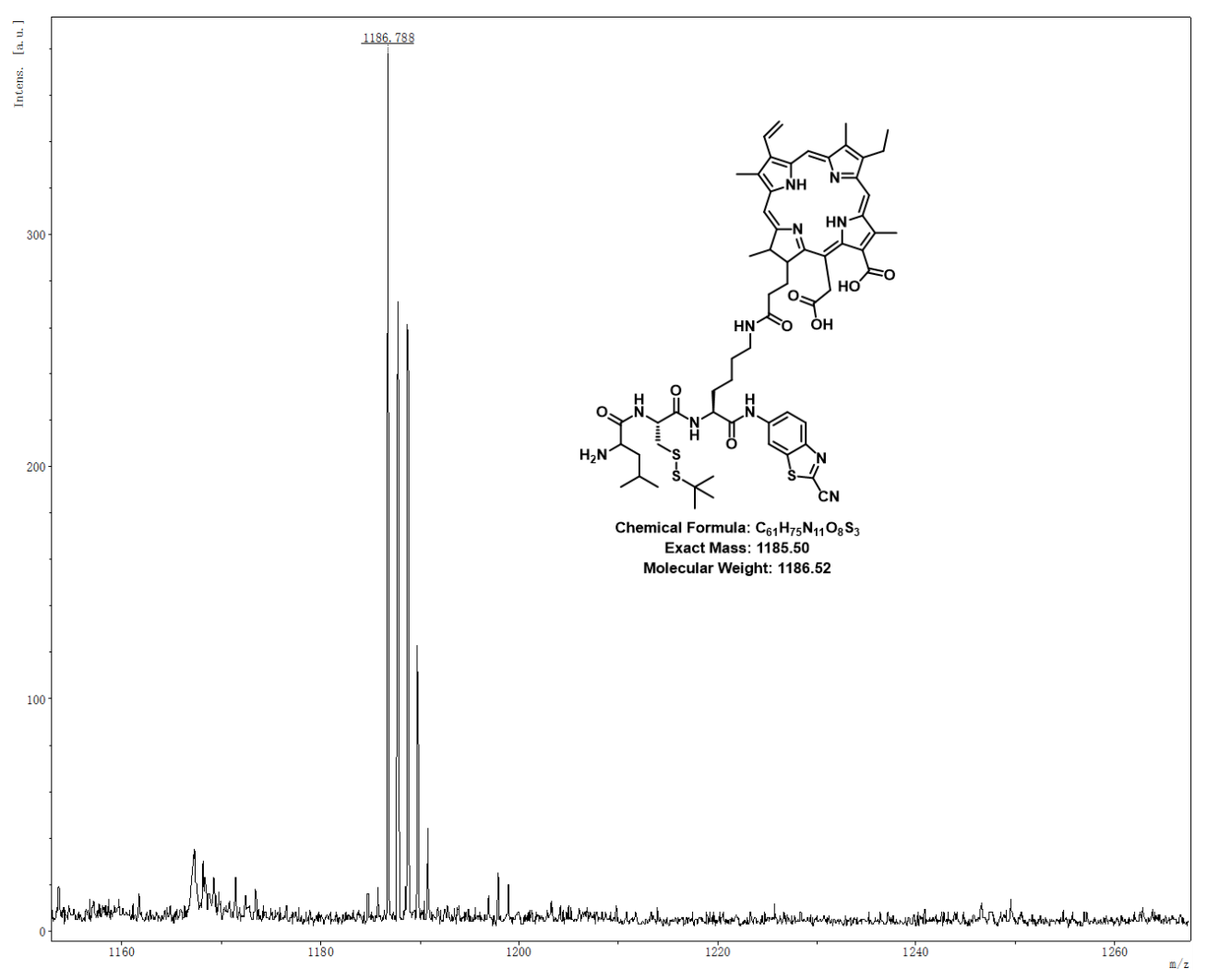

Figure S6. MALDI-TOF/MS spectrum of compound Ce6-Leu.

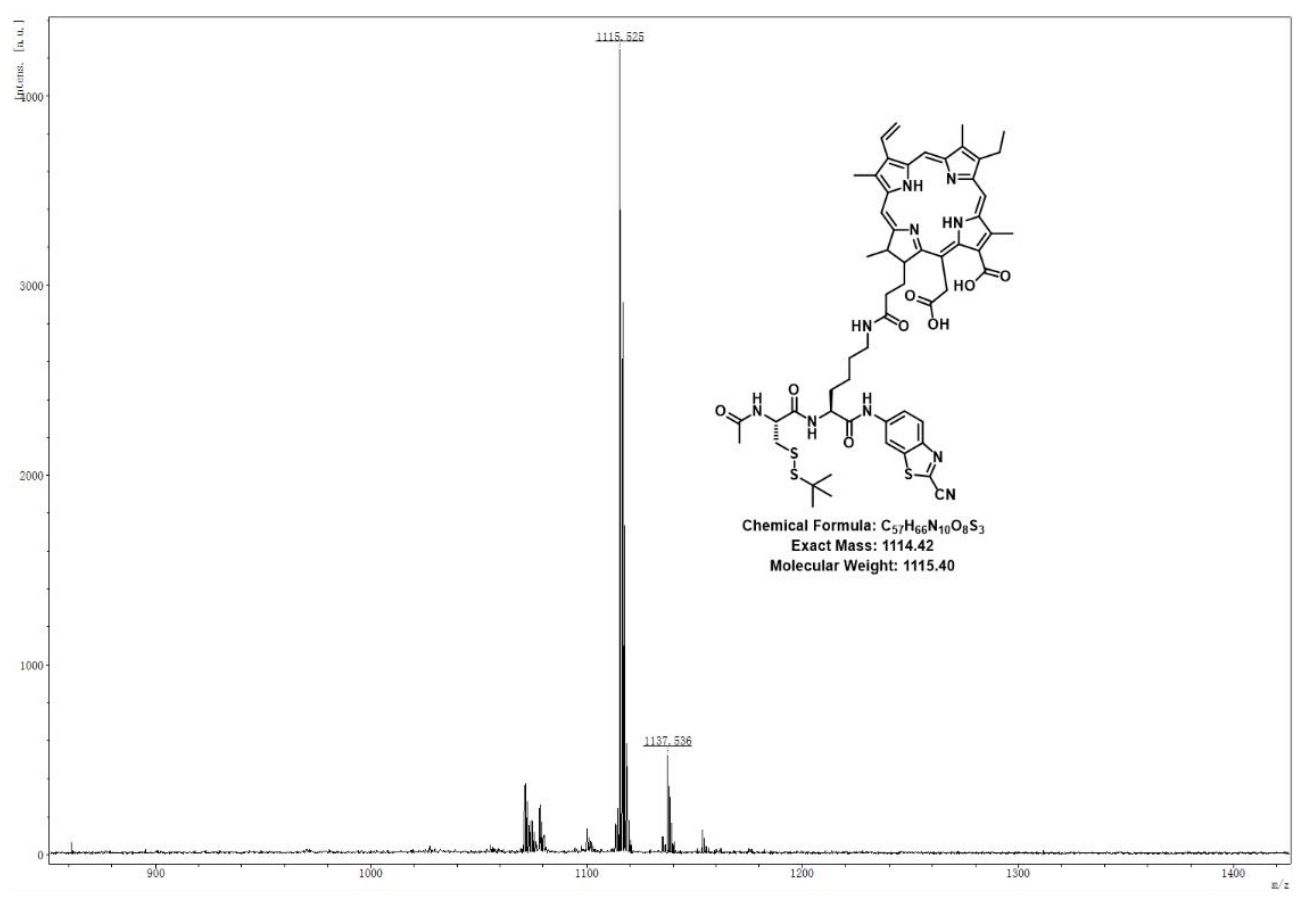

Figure S7. MALDI-TOF/MS spectrum of compound Ce6-Ac. 

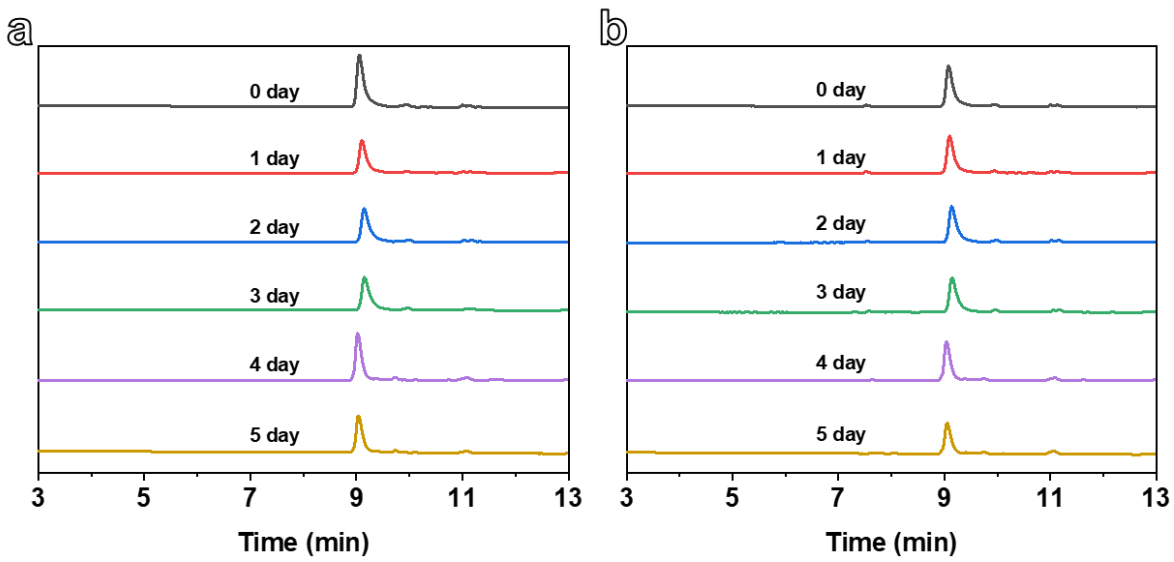

Figure S8. HPLC spectra of probe Ce6-Leu in (a) 10\% serum-containing buffer and (b) acidic buffer $(\mathrm{pH}=5.5)$ for 5 days.

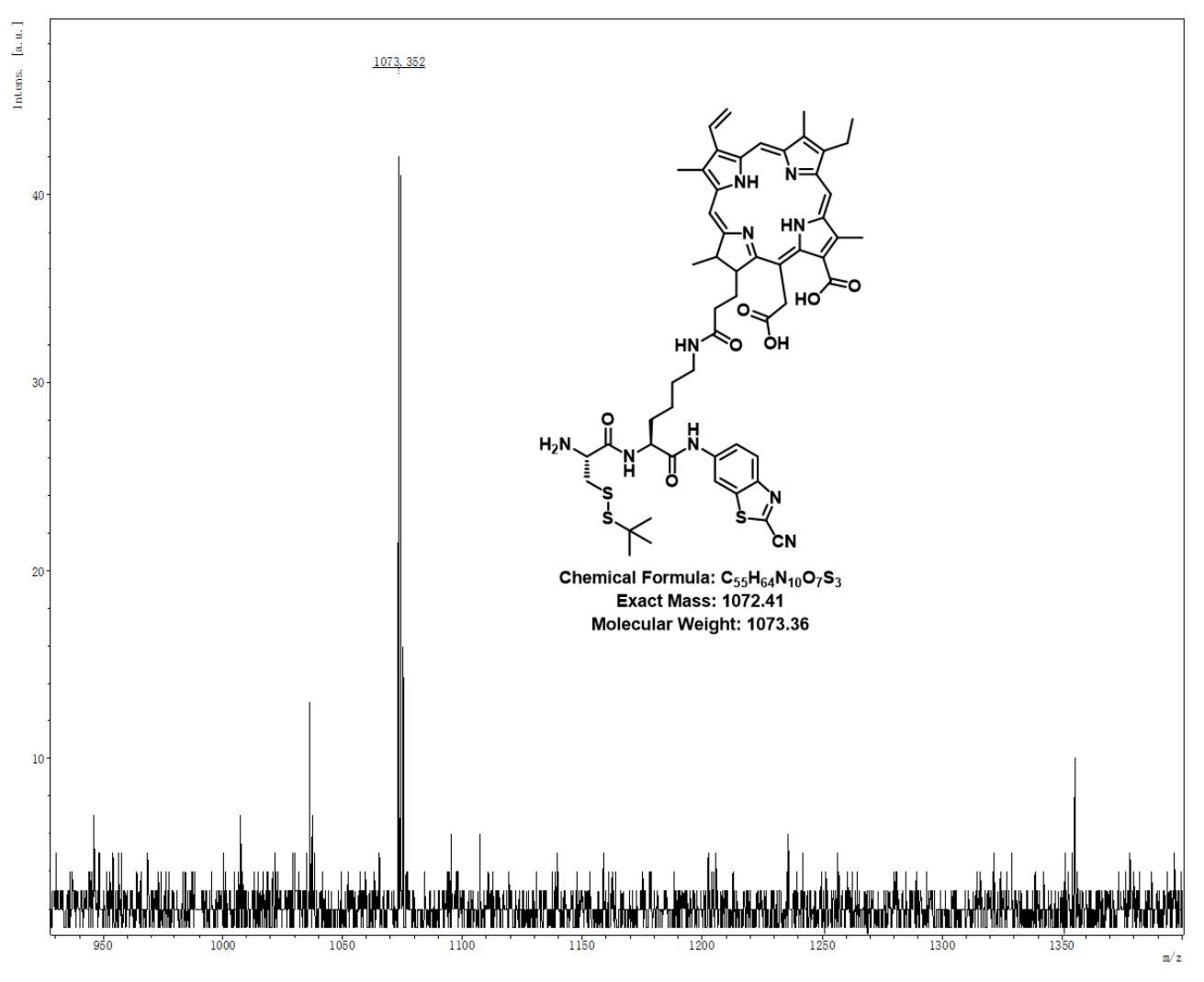

Figure S9. MALDI-TOF/MS spectrum of compound Ce6-Cleaved. 


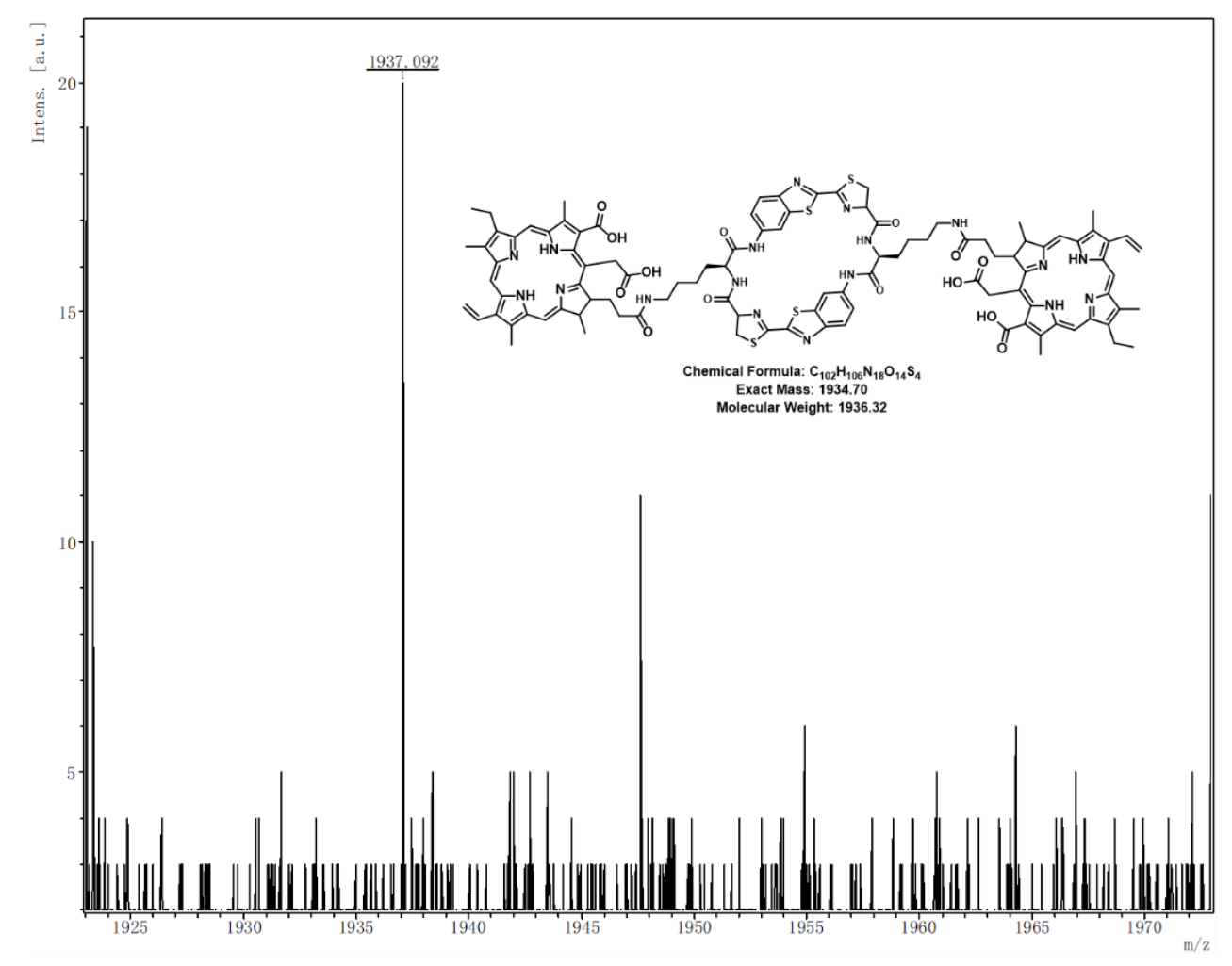

Figure S10. MALDI-TOF/MS spectrum of compound Ce6-dimer.
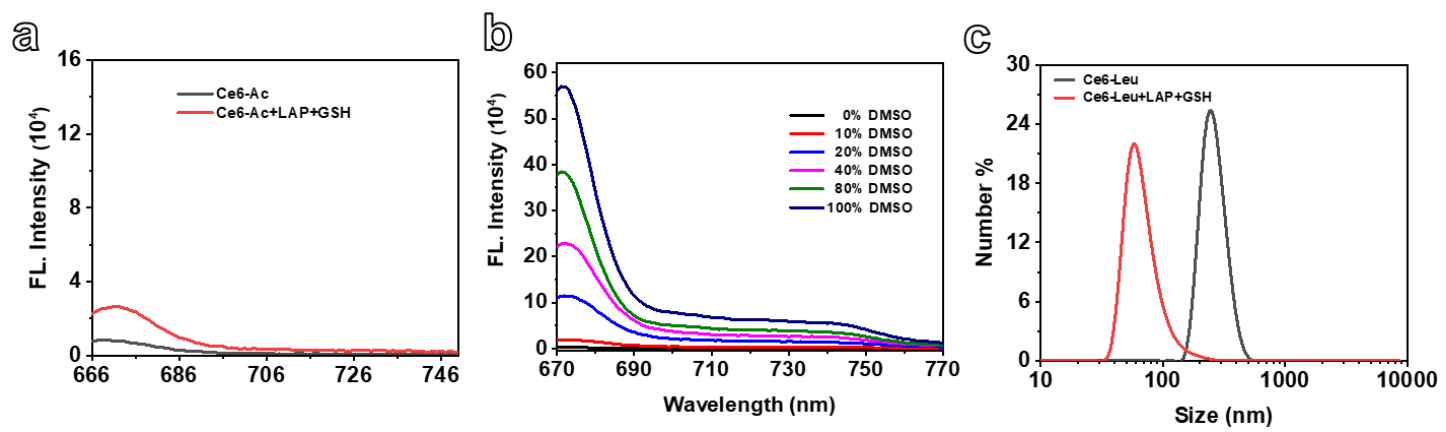

Figure S11. (a) Fluorescence spectrum of Ce6-Ac (black) and Ce6-Ac treated with LAP and GSH (red). (b) Fluorescence spectrum of Ce6-Leu in DMSO/water mixtures. (c) Size distribution of Ce6-Leu (black) and Ce6-Leu treated with LAP and GSH (red) measured by DLS. 


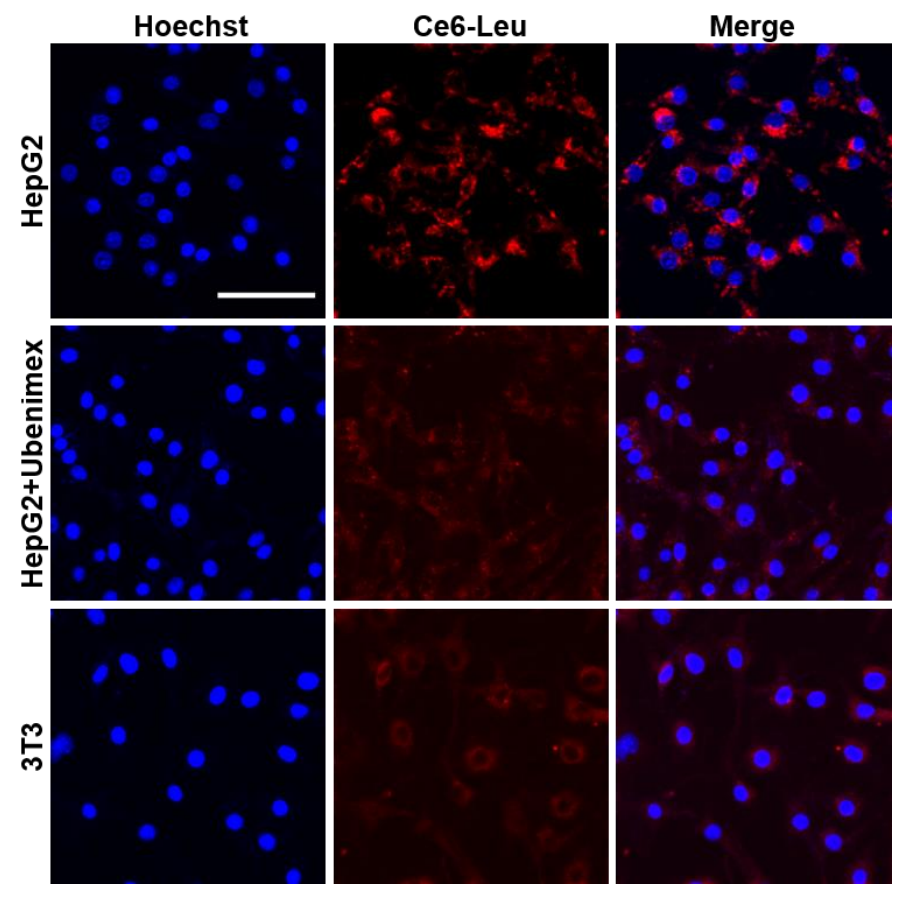

Figure S12. Fluorescence imaging of 3 T3 cells, HepG2 cells incubated with Ce6-Leu and HepG2 cells pretreated with the LAP inhibitor Ubenimex $(50 \mu \mathrm{M})$ for $1 \mathrm{~h}$ and then further incubated with Ce6-Leu. scale bar: $60 \mu \mathrm{m}$

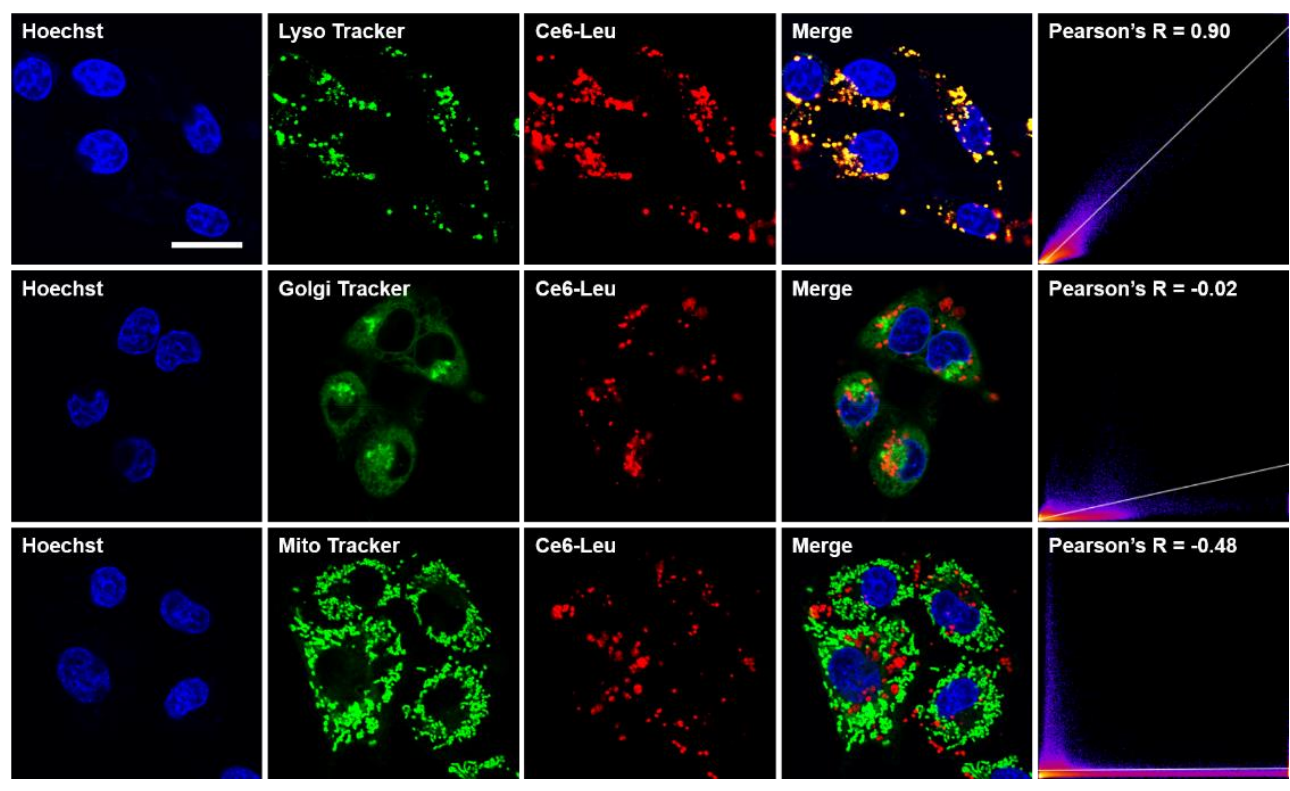

Figure S13. Confocal microscopy images of Ce6-Leu-pretreated HepG2 cells treated with Lyso tracker, Golgi tracker or Mito tracker. scale bar: $20 \mu \mathrm{m}$ 


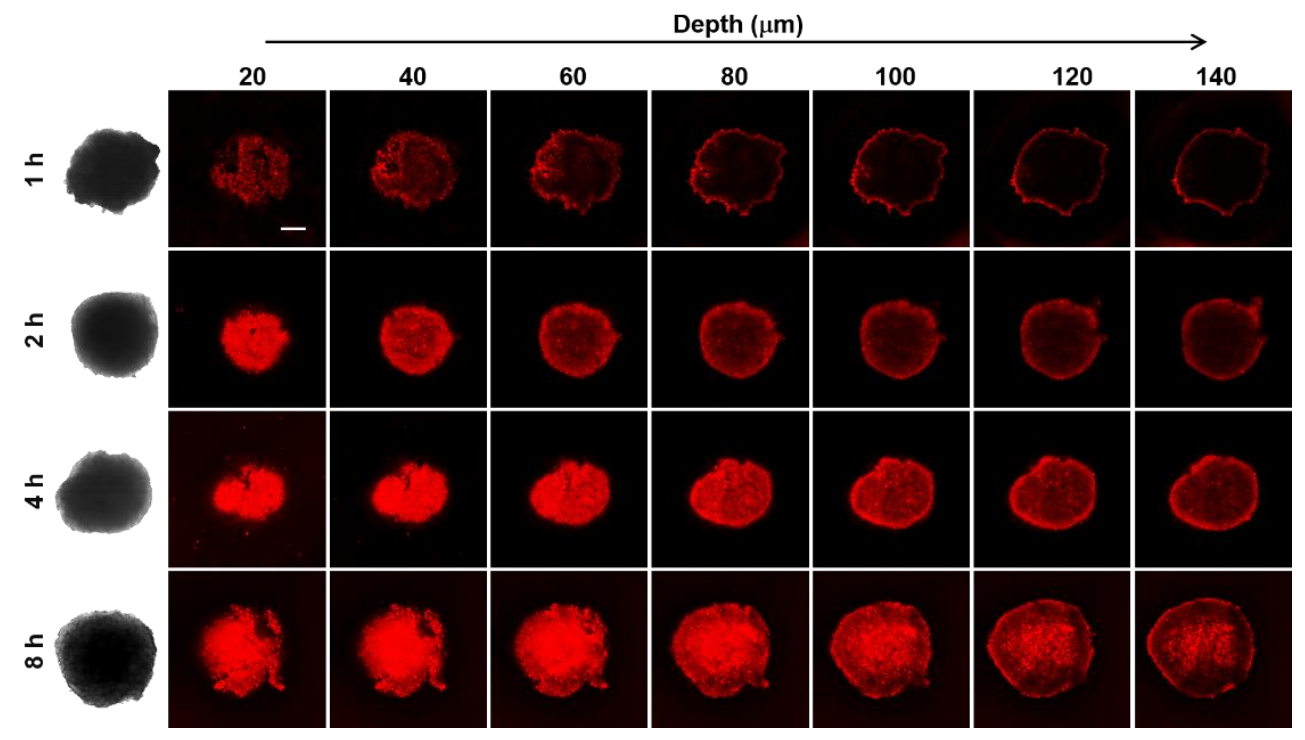

Figure S14. Z-stack CLSM images of HepG2-tumor cell spheroids treated with Ce6-Leu at different times. scale bar: $200 \mu \mathrm{m}$

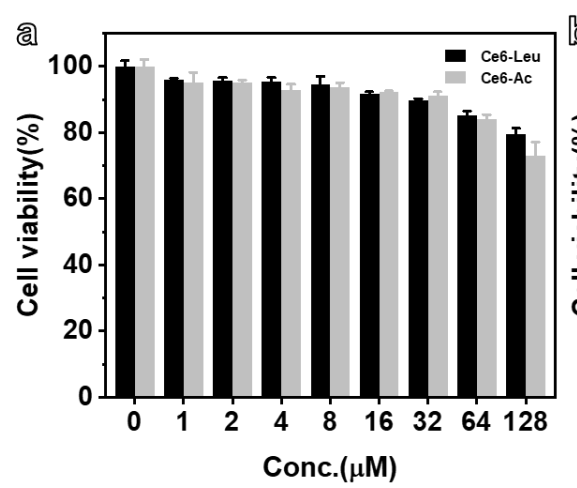

Figure S15. Cell viability of $3 \mathrm{~T} 3$ (a) and HepG2 (b) cells incubated with different concentrations of Ce6-Leu and Ce6-Ac for $24 \mathrm{~h}$.

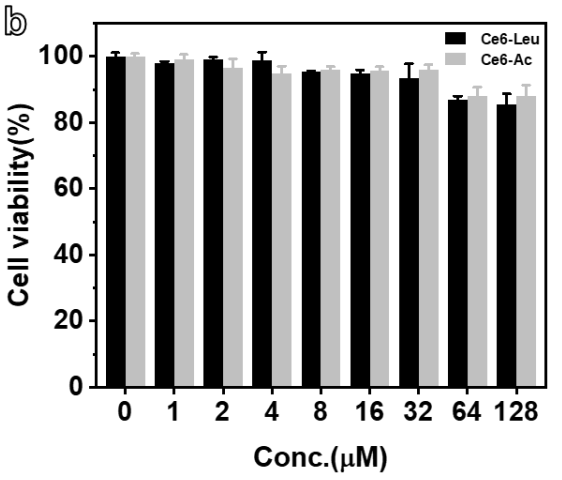

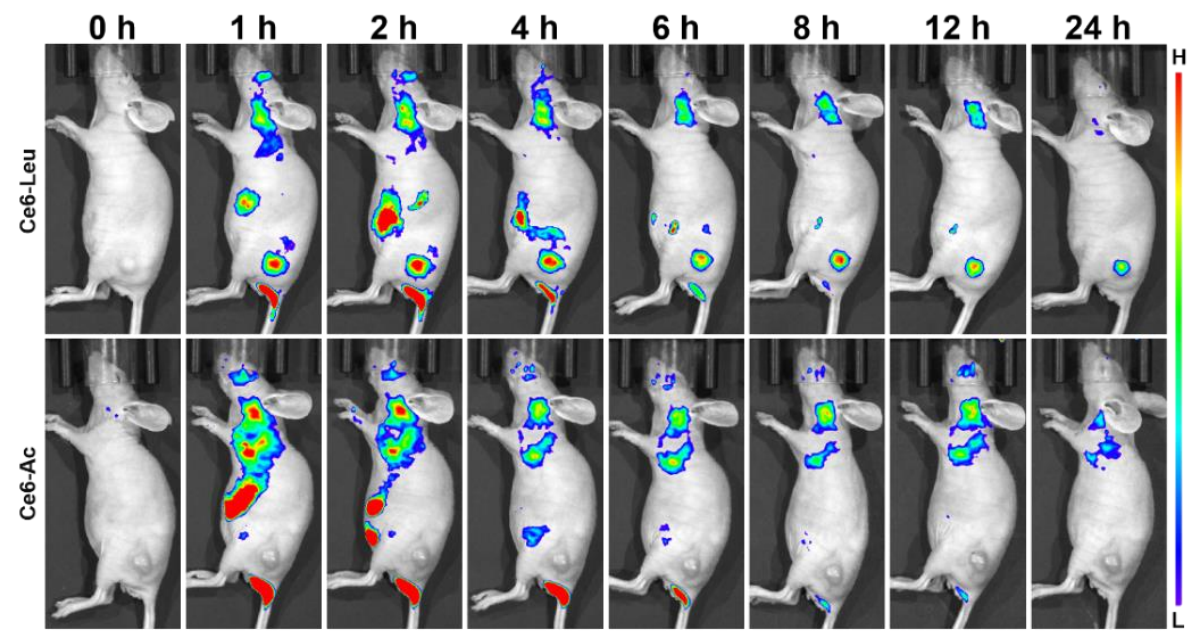

Figure S16. In vivo NIRF images of the mice bearing HepG2 tumor at different time after tail intravenous injection of Ce6-Leu or Ce6-Ac (100 $\mu \mathrm{M}, 200 \mu \mathrm{L})$. 

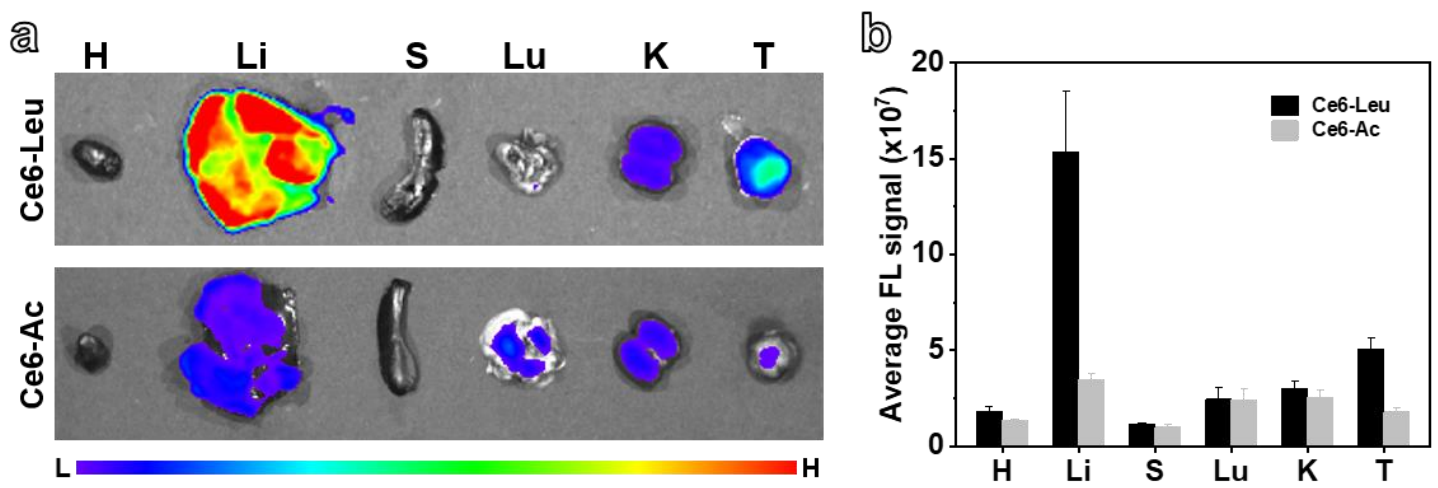

Figure S17. (a) Fluorescence images and (b) quantitative biodistribution analysis of major organs (heart, liver, spleen, lung, kidney, tumor termed as $\mathrm{H}, \mathrm{Li}, \mathrm{S}, \mathrm{Lu}, \mathrm{K}, \mathrm{T}$ ) taken from the mice injected with probe for 4 hours in each group.

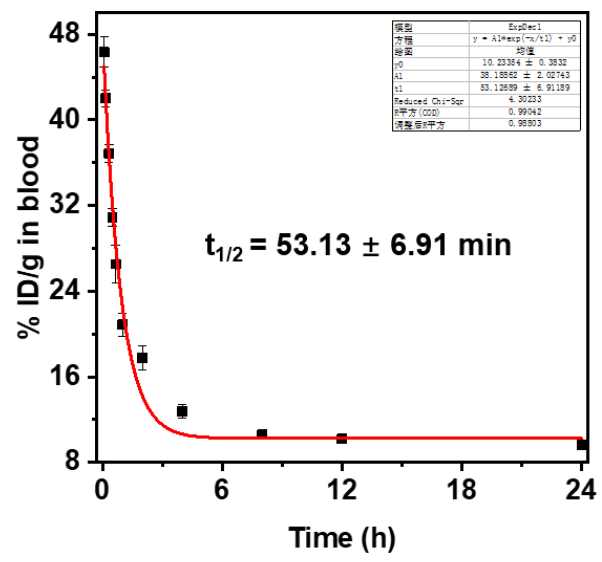

Figure S18. The blood circulation curve of Ce6-Leu after intravenous injection.

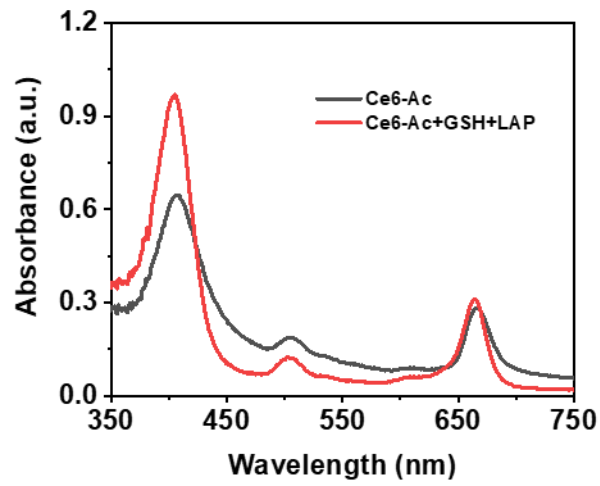

Figure S19. UV-vis absorbance spectra of Ce6-Ac (black) and Ce6-Ac treated with LAP and GSH (red). 

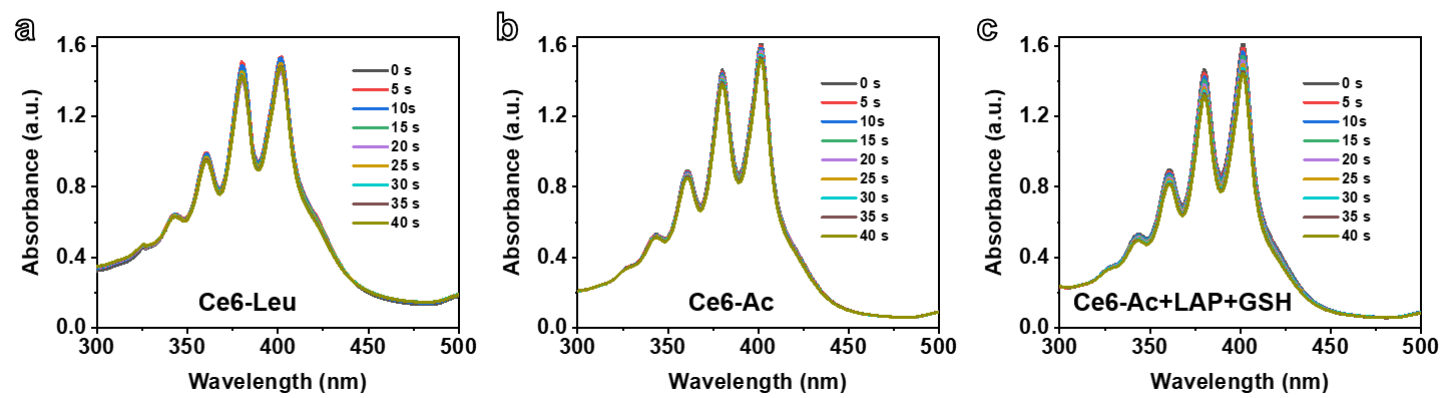

Figure S20. Time-dependent UV-Vis absorption spectra of ABDA oxidation by ${ }^{1} \mathrm{O}_{2}$ radicals generated by the solution of (a) Ce6-Leu, (b) Ce6-Ac and (c) Ce6-Ac treated with LAP and GSH.

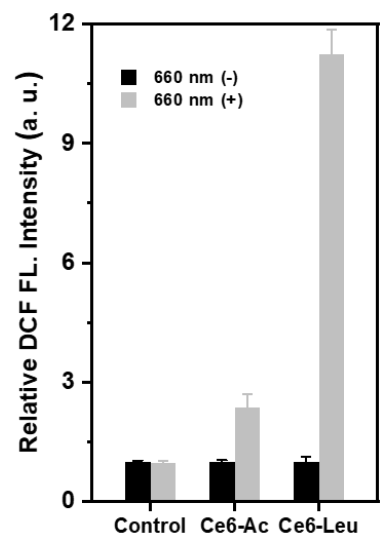

Figure S21. Quantitative analysis carried out by Microplate System of ${ }^{1} \mathrm{O}_{2}$ generation after treating HepG2 cells with PBS, PBS plus laser, Ce6-Leu, Ce6-Leu plus laser, Ce6-Ac, and Ce6-Ac plus laser.

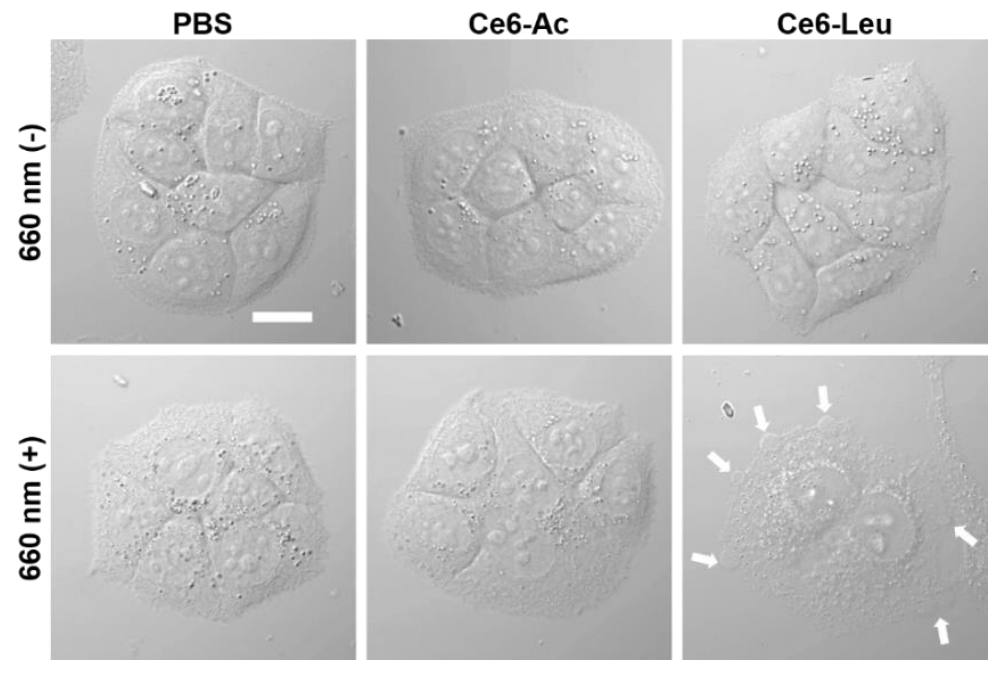

Figure S22. DIC images of HepG2 cells after different experimental operations. The white arrows indicate membrane blebs. scale bar: $15 \mu \mathrm{m}$ 


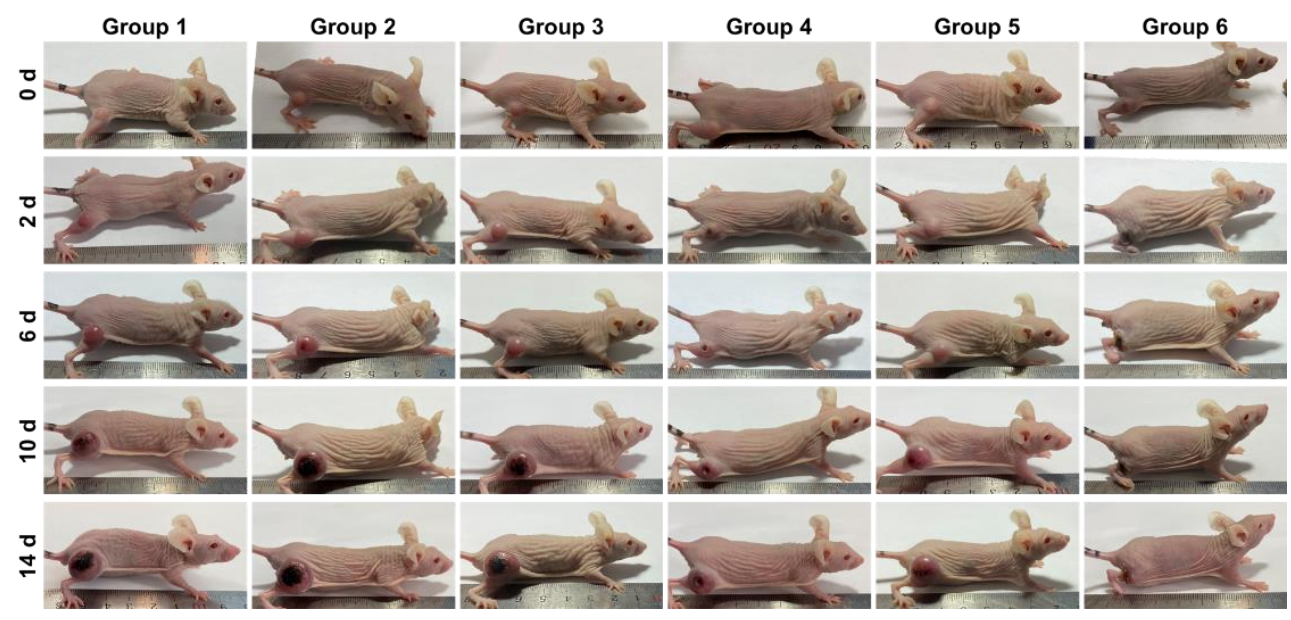

Figure S23. Photographs of representative mice captured on day $0,2,6,10$ and 14 posttreatment.

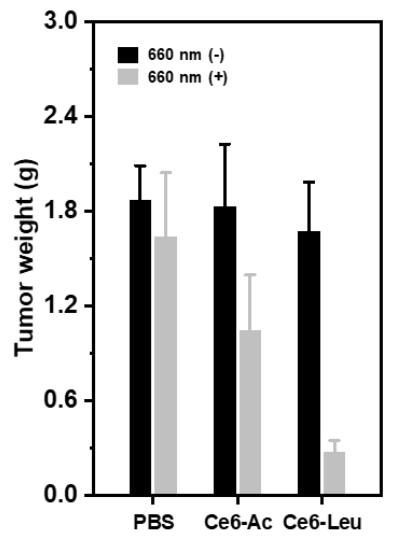

Figure S24. The average weight of various groups tumors after 14 days of different treatments.

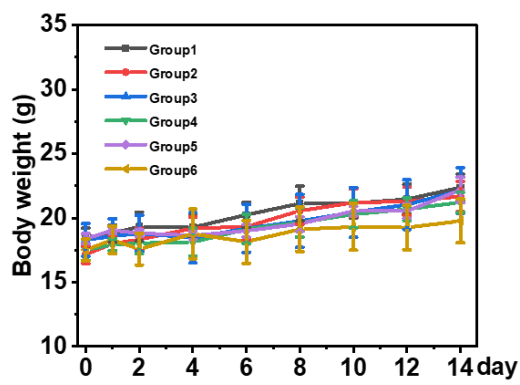

Figure S25. Average body weights of mice 14 days after the treatments. 


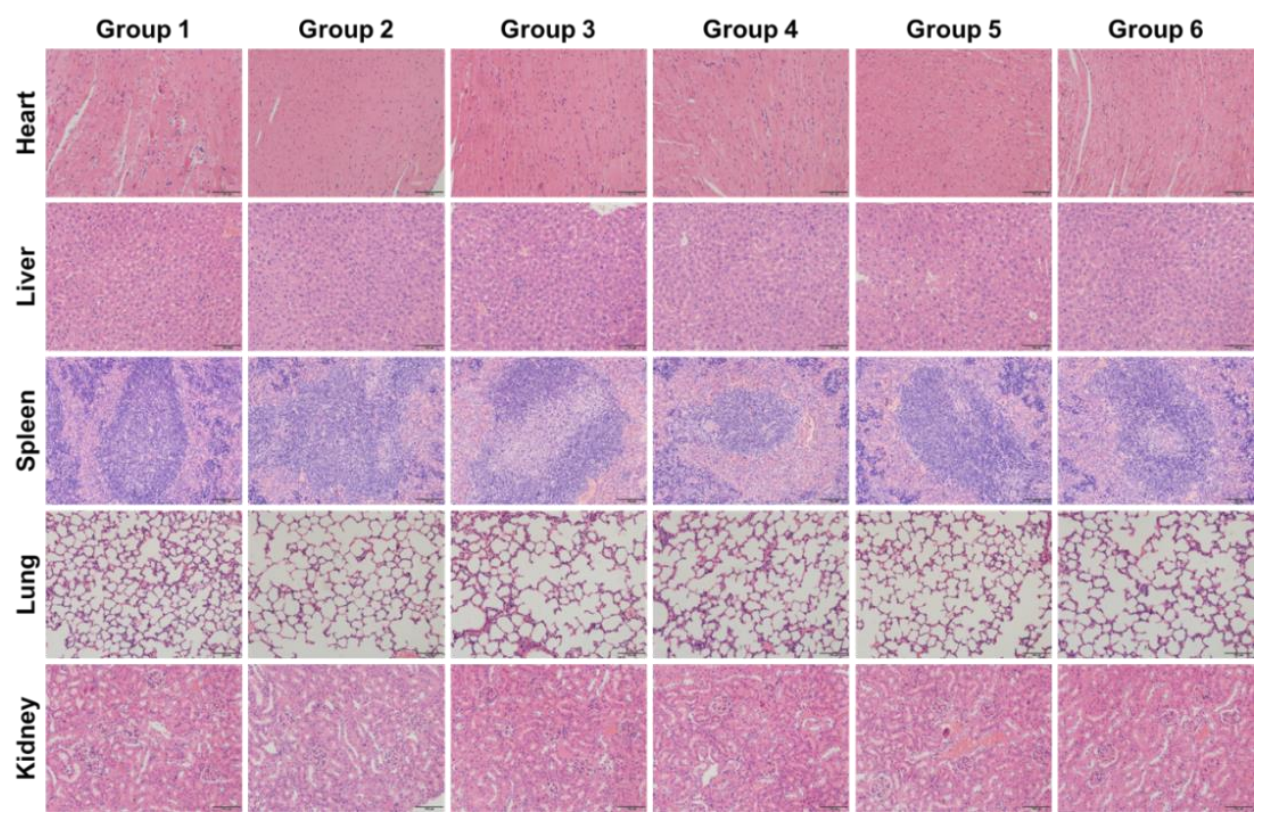

Figure S26. Slices of the major organs harvested from tumor-bearing mice receiving different treatments, stained hematoxylin and eosin (H\&E).

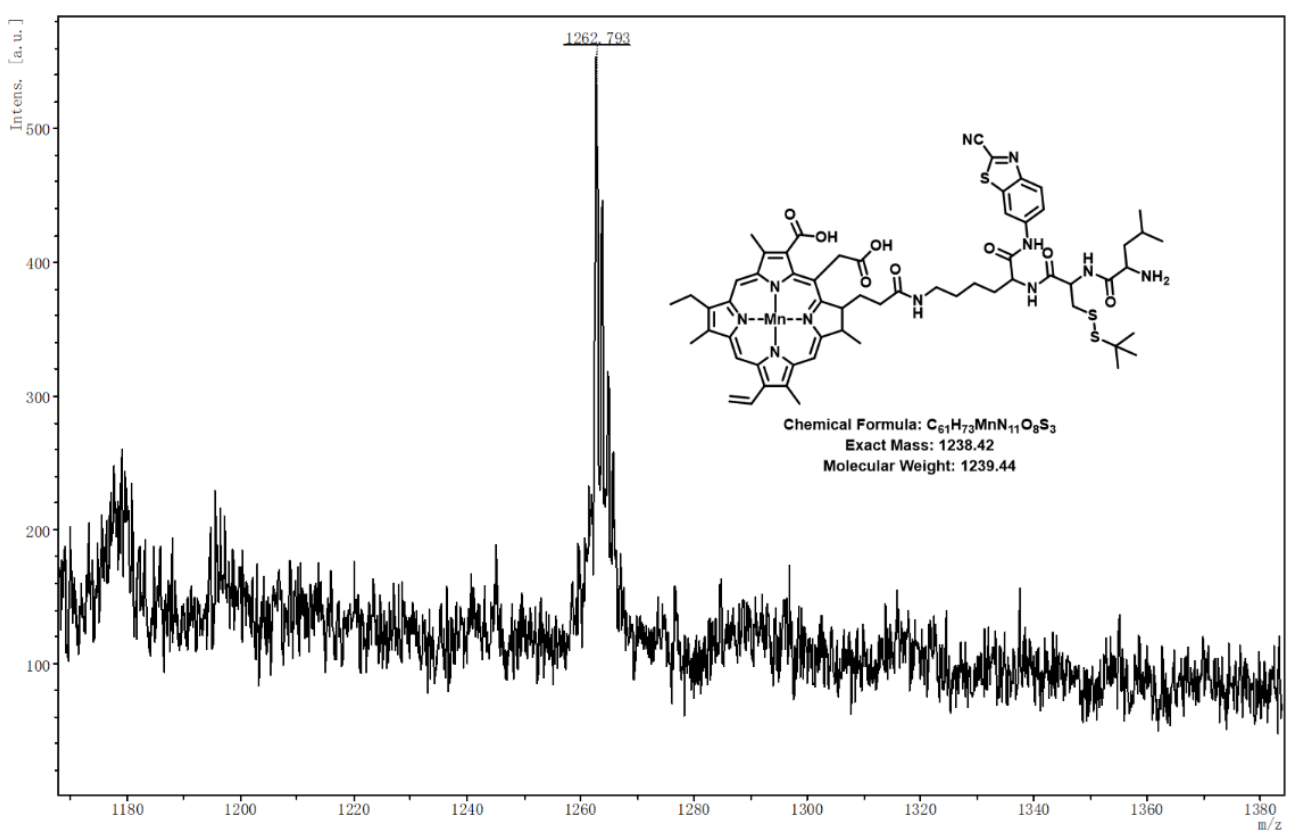

Figure S27. MALDI-TOF/MS spectrum of compound Ce6-Leu@Mn²+. 


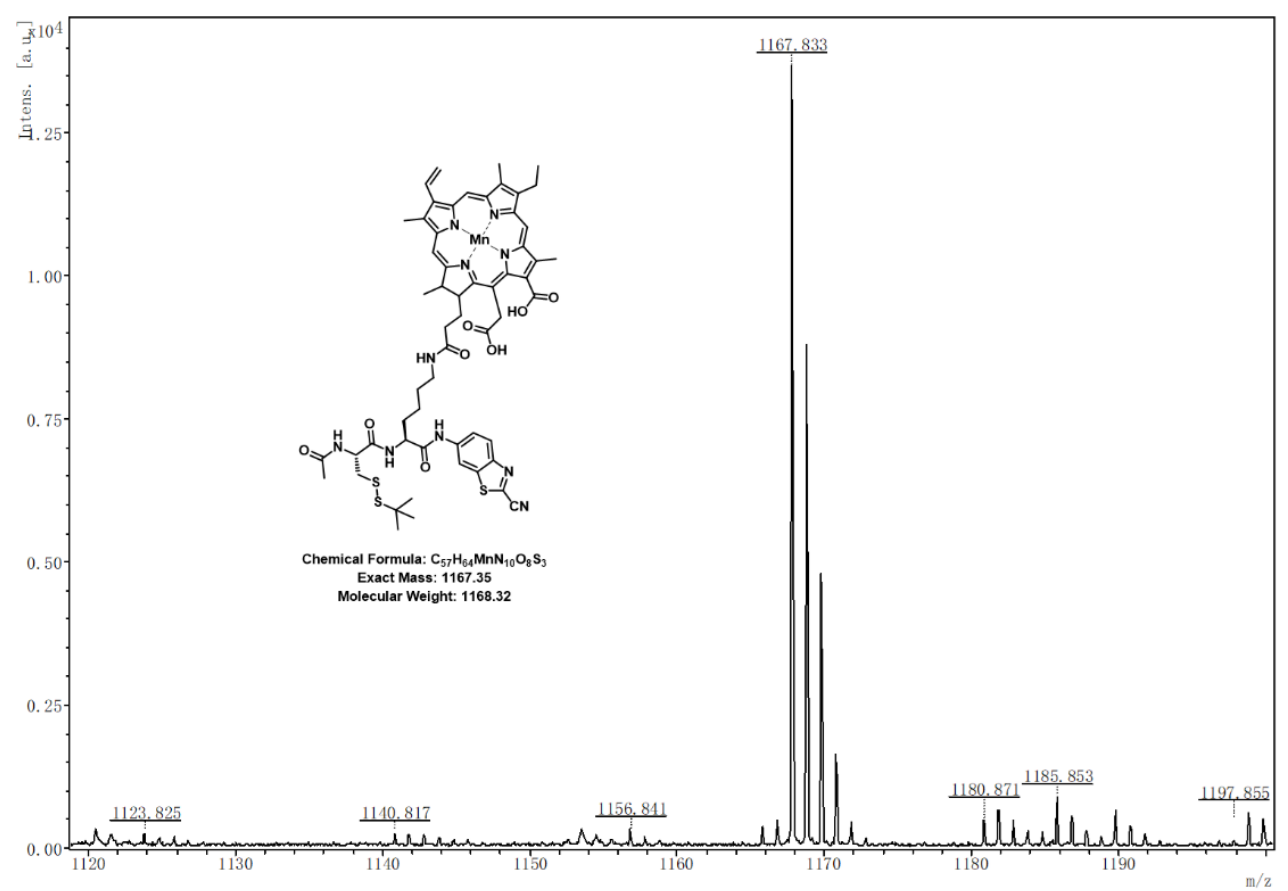

Figure S28. MALDI-TOF/MS spectrum of compound Ce6-Ac@Mn²+.

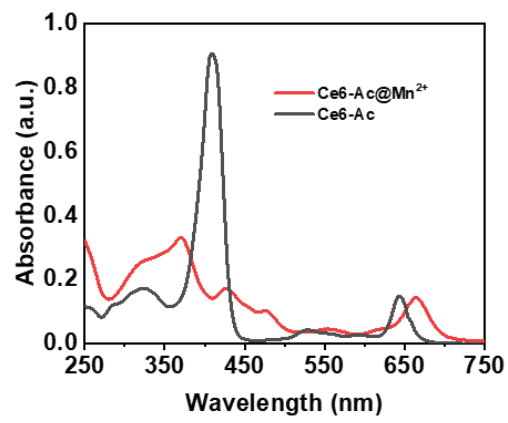

Figure S29. UV-vis absorbance spectra of Ce6-Ac and $\mathrm{Mn}^{2+}$ chelated Ce6-Ac (Ce6$\left.\mathrm{Ac} @ \mathrm{Mn}^{2+}\right)$.

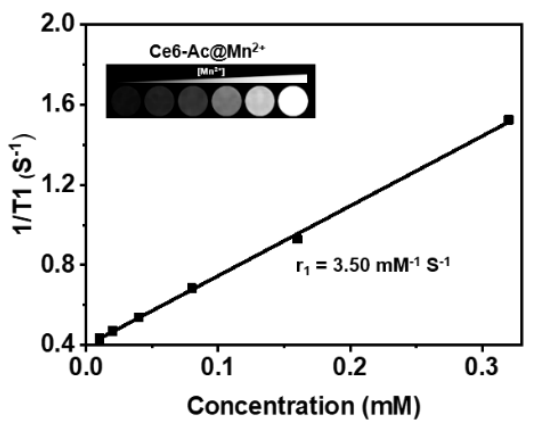

Figure S30. T1-weighted MR images and longitudinal relaxivity $\left(r_{1}\right)$ of Ce6-Ac@Mn²+ 


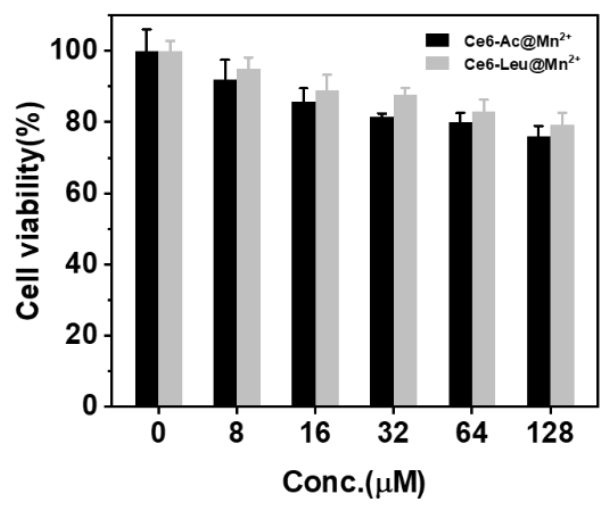

Figure S31. Cell viability of 3T3 cells incubated with different concentrations of Ce6Leu@Mn ${ }^{2+}$ and Ce6-Ac@Mn ${ }^{2+}$ for 24 h.

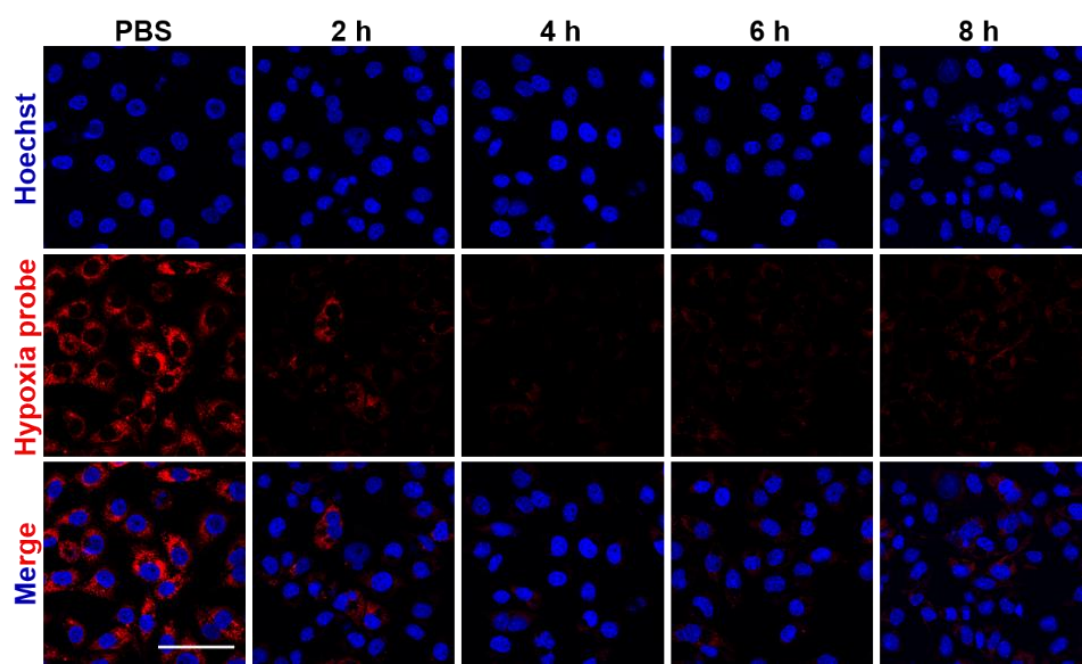

Figure S32. Representative fluorescence images of HpeG2 cancer cells stained with Hoechst 33342 (blue, nuclei) and hypoxia probe (red, hypoxic cells) after incubating with Ce6-Leu@Mn ${ }^{2+}$ with different time. scale bar: $60 \mu \mathrm{m}$

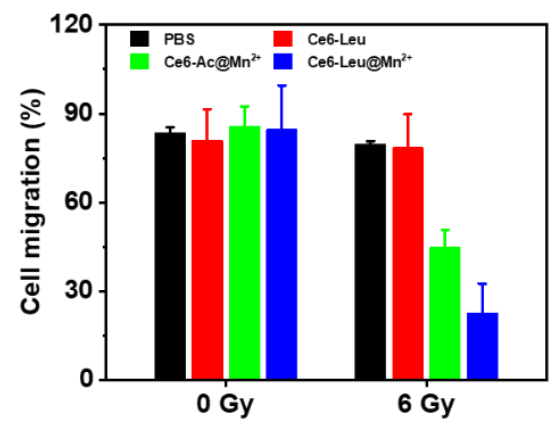

Figure S33. The corresponding quantitative analysis of Cell migration of HepG2 cells treated with different materials under X-ray irradiation ( 0 and $6 \mathrm{~Gy}$ ). 

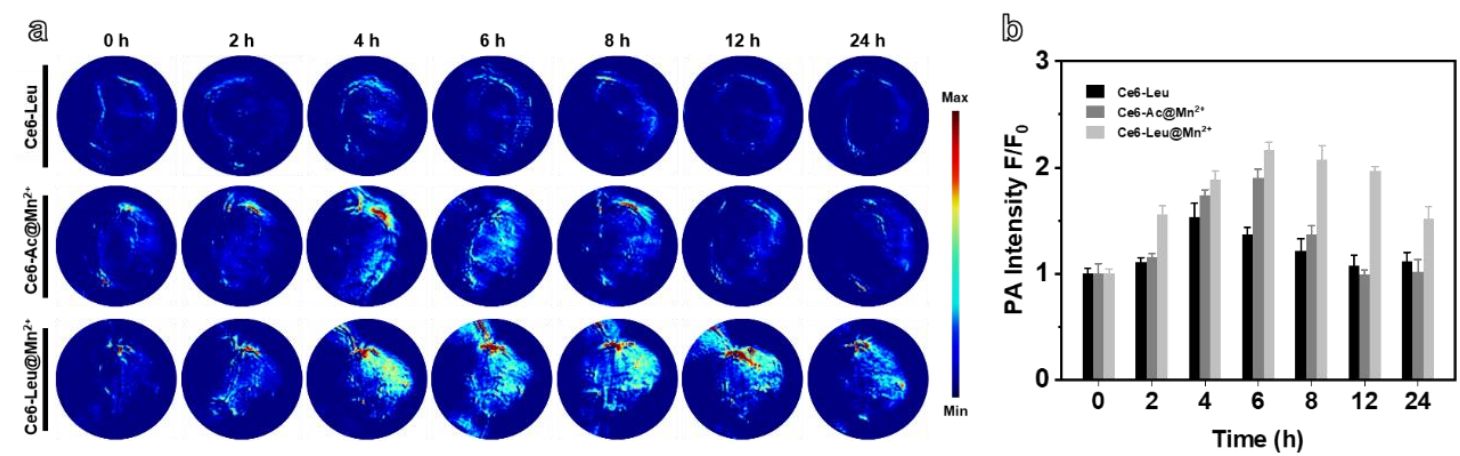

Figure S34. (a) In vivo PA images of the tumor and (b) the PA signals at different time points after intravenous injection of Ce6-Leu, Ce6-Ac@Mn ${ }^{2+}$ or Ce6-Leu@Mn ${ }^{2+}$, respectively.
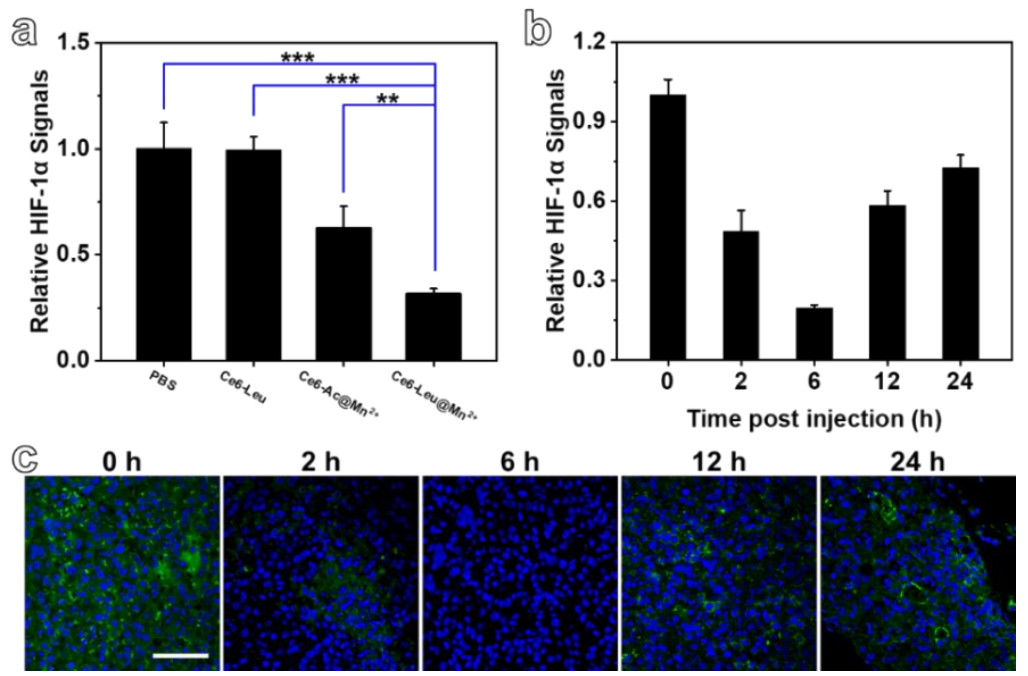

Figure S35. (a) The corresponding quantification of hypoxia inducible factor-1 (HIF-1a) of tumor slices of different groups. ${ }^{* *} \mathrm{P}<0.001$, ${ }^{* *} \mathrm{P}<0.01$. (b) The corresponding immunohistochemical index quantitative analysis and (c) representative HIF-1 $1 \alpha$ staining of tumor sections in different time after tail intravenous injection of Ce6-Leu@Mn²+.

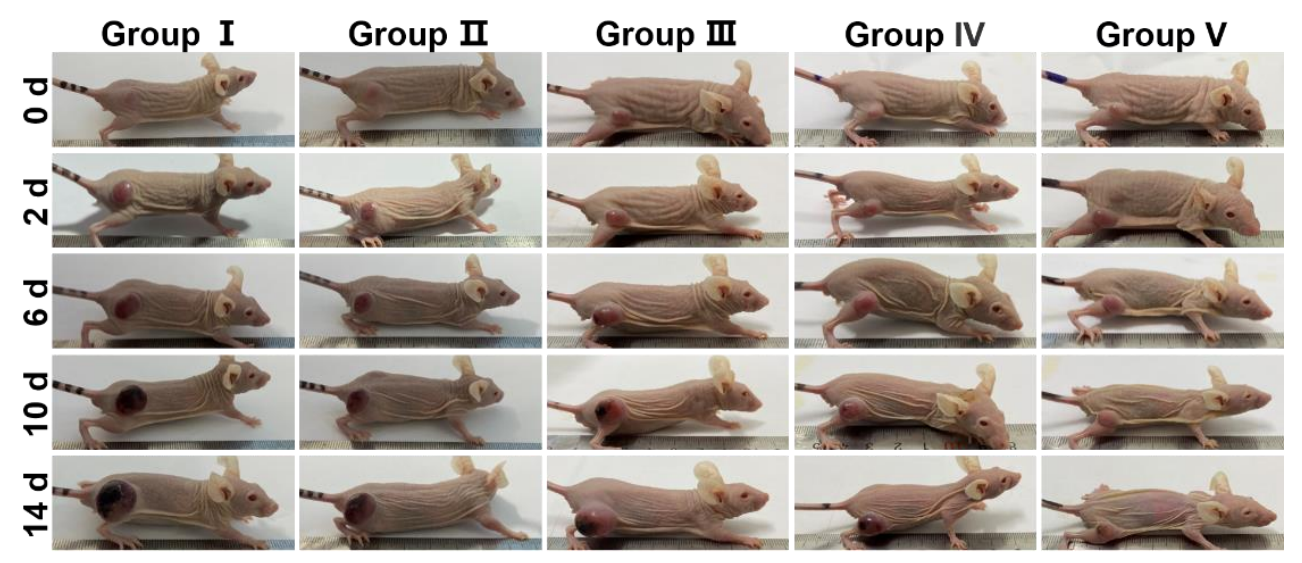

Figure S36. The mice photos of day 0, 2, 6, 10, 14 after different treatment. 

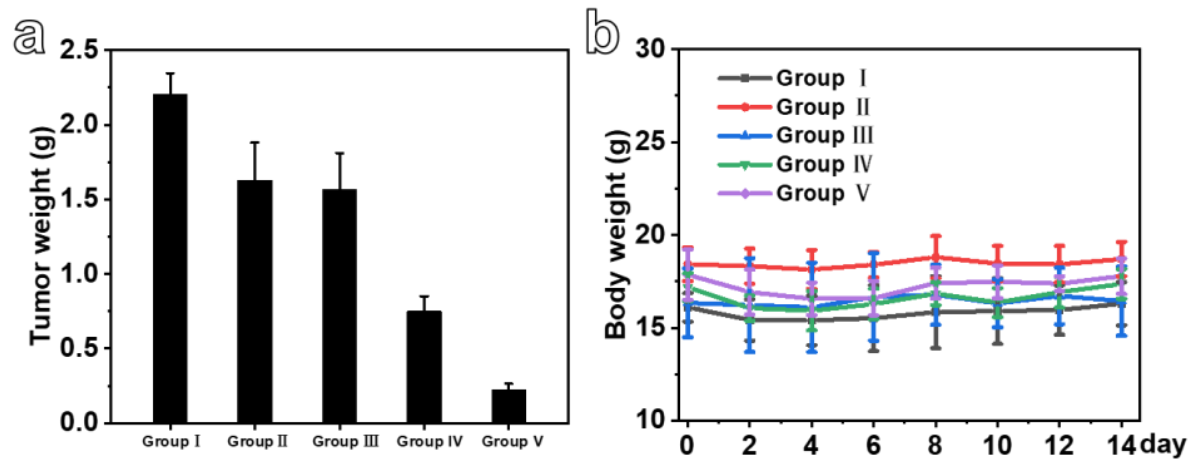

Figure S37. (a) The average weight of various groups tumors after 14 days of different treatments. (b) Average body weights of mice in each group. 